ULB-TH/09-44

TUM-HEP 744/09

DESY 09-221

\title{
Intense Gamma-Ray Lines from Hidden Vector Dark Matter Decay
}

\author{
Chiara Arina ${ }^{a}$, Thomas Hambye ${ }^{a}$, Alejandro Ibarra ${ }^{b}$, Christoph Weniger ${ }^{c}$ \\ a Service de Physique Théorique, \\ Université Libre de Bruxelles, 1050 Brussels, Belgium \\ b Physik-Department T30d, Technische Universität München, \\ James-Franck-Straße, 85748 Garching, Germany \\ c Deutsches Elektronen-Synchrotron DESY, Hamburg \\ Notkestraße 85, 22603 Hamburg, Germany
}

\begin{abstract}
Scenarios with hidden, spontaneously broken, non-abelian gauge groups contain a natural dark matter candidate, the hidden vector, whose longevity is due to an accidental custodial symmetry in the renormalizable Lagrangian. Nevertheless, non-renormalizable dimension six operators break the custodial symmetry and induce the decay of the dark matter particle at cosmological times. We discuss in this paper the cosmic ray signatures of this scenario and we show that the decay of hidden vector dark matter particles generically produce an intense gamma ray line which could be observed by the Fermi-LAT experiment, if the scale of custodial symmetry breaking is close to the Grand Unification scale. This gamma line proceeds directly from a tree level dark matter 2-body decay in association with a Higgs boson. Within this model we also perform a determination of the relic density constraints taking into account the dark matter annihilation processes with one dark matter particle in the final state. The corresponding direct detection rates can be easily of order the current experimental sensitivities.
\end{abstract}




\section{Introduction}

One of the most striking features of the dark matter (DM) particle is its longevity at cosmological scales. This fact could be accommodated ad hoc by imposing a new symmetry (discrete or continuous) which prevents the decay of the dark matter particle, such as the $R$-parity in the Minimal Supersymmetric Standard Model or the $Z_{2}$ symmetry assumed in many phenomenological models. The longevity of the dark matter particle is more elegantly explained, however, if it arises as the result of an accidental symmetry of the Lagrangian, in complete analogy to the longevity of the proton, which in the Standard Model framework is explained by being the proton the lightest particle carrying baryon number. A simple implementation of this idea consists in extending the Standard Model gauge group with a non-abelian gauge symmetry, under which all the Standard model particles are singlets, which is spontaneously broken by the vacuum expectation value of a standard model singlet scalar particle. The renormalizable part of the Lagrangian of this model presents an accidental custodial symmetry which prevents the decay of the hidden vector bosons, thus predicting the existence of a particle with the correct dark matter properties [1, 2].

The simplest example of such class of models introduces in the hidden sector an extra $S U(2)_{\text {HS }}$ gauge group plus a complex scalar doublet of this gauge symmetry, which acquires a vacuum expectation value. After the $S U(2)_{\text {HS }}$ spontaneous symmetry breaking, the renormalizable part of the Lagrangian presents a $S O(3)$ custodial symmetry which makes the three components of the $S U(2)_{\text {HS }}$ vector boson degenerate in mass and stable. For wide ranges of the parameters of the model, the relic abundance of the vector multiplets can reproduce the observed dark matter abundance. Furthermore, these parameters are also consistent with the present constraints from electroweak precision measurements and from direct dark matter searches.

Being the $S O(3)$ custodial symmetry an accidental symmetry it is plausibly broken explicitly by higher dimensional operators in the Lagrangian. This is again in complete analogy with the baryon number violating dimension six operators that necessarily appear in the Standard Model Lagrangian, unless the baryon number conservation arises as a residual symmetry of an underlying gauge group. Indeed, there are dimension six operators which violate the custodial symmetry which can induce the decay of the dark matter particle, whereas analogous dimension five operators are absent The scale of custodial symmetry breaking has a lower bound stemming from the requirement that the dark matter lifetime has to be longer than the age of the Universe, $\tau_{\mathrm{DM}} \gtrsim 10^{17} \mathrm{~s}$.

\footnotetext{
${ }^{1}$ In this respect, to consider an accidental symmetry is different from justifying the dark matter stability from a residual discrete subgroup of a Grand Unification gauge group [3, 4, 5, 6, 7, 8, where the latter forbids any decay.
} 
Furthermore, the dark matter decay produces a flux of stable particles, such as positrons, antiprotons and gamma rays. The requirement that the exotic flux of cosmic rays does not exceed the measured fluxes translates into a more stringent constraint on the dark matter lifetime and in turn on the scale of custodial symmetry breaking.

On the other hand, a series of experiments measuring high-energy charged cosmic rays have recently reported strong indications for the existence of an excess of positrons at high energies. Namely, the PAMELA measurements of the positron fraction show an energy spectrum which rises steeply at energies 7-100 GeV, possibly extending towards higher energies [9], while the secondary positron flux calculated from state-of-the-art propagation models [10], together with the total electron plus positron flux measured by the Fermi collaboration [11], predict a positron fraction which decreases monotonically with the energy. Furthermore, the electron plus positron flux measured by Fermi is harder than expected from conventional diffusive models [12], also suggesting the existence of an excess of electrons and positrons at higher energies, with a cut-off at around $1 \mathrm{TeV}$ as observed by the H.E.S.S. collaboration [13, 14]. It is interesting to mention that one possible explanation for the electron/positron excesses is precisely the decay of dark matter particles in the Milky Way halo with a mass in the TeV range and a lifetime $\sim 10^{26} \mathrm{~s}$ [15, 16]. Nevertheless, irrespectively of the origin of the electron/positron excesses, these measurements set a constraint on the exotic flux of electrons/positrons. Furthermore, models of dark matter decay are severely constrained by the PAMELA measurements of the antiproton-to-proton ratio [17], which does not show a deviation from the expectations of conventional production mechanism. Interestingly, for $\mathrm{TeV}$ mass dark matter particles which decay via dimension six operators, this value for the lifetime naturally arises if the dimension six operators are suppressed by a mass scale close to the scale of grand unification [18, 19, 20, 21, 22, 23]. Therefore, present cosmic ray observations provide a way of testing some scenarios of Grand Unification.

One of the well-known smoking gun signals for dark matter in the sky is the possible observation of a sharp gamma-ray line [24, 25, 26]. The $\gamma \gamma$ and $\gamma Z$ lines have been exhaustively studied in supersymmetric models, for the neutralino dark matter [27, 28, 29, 30, 31, 32], in the inert doublet model [33] and for Kaluza-Klein dark matter [34. Recently it has been pointed out that a monochromatic gamma line can be also produced accompanied to an Higgs boson [35]. Note that the gamma lines in all these models are induced by annihilation of dark matter particles at one loop level (for an exception see Ref. [36]). However, in many situations the disentanglement of the gamma lines from the background requires either a dark matter mass in the $\mathrm{TeV}$ range or astrophysical boost factors to make the signal strong enough. On the other hand, intense gamma-ray lines can also appear at tree level in the decay of dark matter gravitinos in supersymmetric 
scenarios where R-parity conservation is not imposed [37, 38, 39, 40, 41]. In both cases, the observation of a gamma-ray line would be a very clean indirect detection signature for annihilating or decaying dark matter and is a promising signature to search for.

In this paper we will analyze the cosmic ray signatures stemming from the decay of a hidden $S U(2)$ vector boson. A decay, unlike most annihilations processes, does not require any "boost factor" in order to lead to cosmic ray rates well above backgrounds. Moreover it leads to larger extragalactic fluxes. We will work out the possible decay modes in detail and concentrate on gamma-ray line and anti-matter signatures. Most interestingly, we find that hidden vector dark matter decay modes with gamma-ray lines in the final state are automatically present already at tree level. These decay modes exist for every possible dimension six operator leading to the hidden vector dark matter decay and are hence a robust prediction of the model. Taking advantage of the fact that the dark matter has spin-1, the gamma lines arise directly at tree level from DM decays to $\gamma h$ or $\gamma \eta$ where $h$ and $\eta$ are the standard model and hidden sector Higgs boson respectively. Furthermore, one of the operators generates a kinetic mixing between hidden sector and the hypercharge $U(1)_{Y}$ giving rise to two-body decay modes into charged leptons. We will discuss these properties in light of recent and future cosmic-ray observations.

The paper is organized as follows: in section 2 we will introduce the hidden vector dark matter model and discuss the possible decay modes in detail. In section 3 we will discuss these decay modes for the different operators separately and show results for several benchmark models, giving particular emphasis to gamma-ray line signatures. In section 4 we improve the calculation of the relic density taking into account the dark matter annihilations with one DM particle in the final state, and compute the direct detection cross sections it gives. We draw conclusions in section 5 .

\section{Hidden $S U(2)$ model with custodial symmetry breaking}

We consider an extension of the Standard Model where the gauge group contains a hidden non-abelian group, $S U(2)_{\mathrm{HS}}$, with gauge bosons $A^{\mu}$. We assume that this symmetry is spontaneously broken via the vacuum expectation value of a complex $S U(2)_{\mathrm{HS}}$ doublet scalar field, $\phi$. We further assume that all the Standard Model particles are singlets under $S U(2)_{\mathrm{HS}}$, thus the Standard Model only couples to the hidden sector via the Higgs portal term $|\phi|^{2}|H|^{2}$, being $H$ the Standard Model Higgs doublet (note that the kinetic mixing of the $S U(2)_{\mathrm{HS}}$ gauge multiplet $A^{\mu}$ with the SM gauge bosons is forbidden by the non-abelian character of the extra gauge symmetry). Under these assumptions, 
the renormalizable part of the Lagrangian reads:

$$
\mathcal{L}=\mathcal{L}^{S M}-\frac{1}{4} F^{\mu \nu} \cdot F_{\mu \nu}+\left(\mathcal{D}_{\mu} \phi\right)^{\dagger}\left(\mathcal{D}^{\mu} \phi\right)-\lambda_{m} \phi^{\dagger} \phi H^{\dagger} H-\mu_{\phi}^{2} \phi^{\dagger} \phi-\lambda_{\phi}\left(\phi^{\dagger} \phi\right)^{2},
$$

where $\mathcal{D}^{\mu}=\partial^{\mu} \phi-i \frac{g_{\phi}}{2} \tau \cdot A^{\mu}$, being $\tau^{a}, a=1,2,3$ the generators of the hidden $S U(2)$ gauge group. If $\mu_{\phi}^{2}<0$, the hidden sector scalar field $\phi$ acquires a vacuum expectation value, $v_{\phi}$, and the $S U(2)_{\mathrm{HS}}$ symmetry is broken spontaneously, with $v_{\phi}=\left(-\mu_{\phi}^{2} \lambda+\lambda_{m} \mu^{2} / 2\right) /\left(\lambda \lambda_{\phi}-\right.$ $\left.\lambda_{m}^{2} / 4\right)$. In the unitary $S U(2)_{\text {HS }}$ gauge the Lagrangian of the theory is:

$$
\begin{aligned}
\mathcal{L}= & \mathcal{L}_{S M}-\frac{1}{4} F_{\mu \nu} \cdot F^{\mu \nu}+\frac{1}{8}\left(g_{\phi} v_{\phi}\right)^{2} A_{\mu} \cdot A^{\mu}+\frac{1}{8} g_{\phi}^{2} A_{\mu} \cdot A^{\mu} \eta^{2}+\frac{1}{4} g_{\phi}^{2} v_{\phi} A_{\mu} \cdot A^{\mu} \eta^{\prime} \\
& +\frac{1}{2}\left(\partial_{\mu} \eta^{\prime}\right)^{2}-\frac{\lambda_{m}}{2}\left(\eta^{\prime}+v_{\phi}\right)^{2} H^{\dagger} H-\frac{\mu_{\phi}^{2}}{2}\left(\eta^{\prime}+v_{\phi}\right)^{2}-\frac{\lambda_{\phi}}{4}\left(\eta^{\prime}+v_{\phi}\right)^{4},
\end{aligned}
$$

which gives $M_{A}=g_{\phi} v_{\phi} / 2$ and where $\eta^{\prime}$ is the hidden sector Higgs boson. This Lagrangian has only 4 independent parameters, which can be taken as $g_{\phi}, v_{\phi}, \lambda_{\phi}$ and $\lambda_{m}$.

Once the electroweak sector is broken, the hidden sector $\eta^{\prime}$ mixes with the standard model Higgs boson $h^{\prime}$ through the Higgs portal interaction $\lambda_{m}$

$$
\begin{aligned}
& h^{\prime}=\cos \beta h+\sin \beta \eta, \\
& \eta^{\prime}=-\sin \beta h+\cos \beta \eta .
\end{aligned}
$$

The complete Lagrangian in the $h, \eta$ physical state basis can be found in Ref. [1] as a function of $g_{\phi}, v_{\phi}, \lambda_{\phi}$ and $\lambda_{m}$, together with the corresponding expression for the mixing angle $\beta$.

The Lagrangian in Eq. 2.2 has a remarkable property: it displays a $S O(3)$ custodial symmetry in the $A_{i}^{\mu}$ component space, which prevents any decay to $S O(3)$ singlets (such as Standard Model particles or $\eta^{\prime}$ ). Consequently, if the model is described just by the renormalizable Lagrangian, the three $A_{i}^{\mu}$ components are degenerate in mass and are absolutely stable. Nevertheless, since this $S O(3)$ global symmetry is accidental, one expects in the Lagrangian the existence of non-renormalizable operators suppressed by a large scale $\Lambda$ which break the custodial symmetry. The complete list of operators with dimension smaller or equal than six which lead, after the spontaneous symmetry breaking of $S U(2)_{\mathrm{HS}}$ and $S U(2)_{L} \times U(1)_{Y}$, to the breaking of the $S O(3)$ custodial symmetry reads:

$$
\begin{aligned}
& \text { (A) } \frac{1}{\Lambda^{2}} \mathcal{D}_{\mu} \phi^{\dagger} \phi \mathcal{D}_{\mu} H^{\dagger} H, \\
& \text { (B) } \frac{1}{\Lambda^{2}} \mathcal{D}_{\mu} \phi^{\dagger} \phi H^{\dagger} \mathcal{D}_{\mu} H, \\
& \text { (C) } \frac{1}{\Lambda^{2}} \mathcal{D}_{\mu} \phi^{\dagger} \mathcal{D}_{\nu} \phi F^{\mu \nu Y}, \\
& \text { (D) } \frac{1}{\Lambda^{2}} \phi^{\dagger} F_{\mu \nu}^{a} \frac{\tau^{a}}{2} \phi F^{\mu \nu Y} .
\end{aligned}
$$


In turn, the breaking of the custodial symmetry leads to the decay of the dark matter hidden gauge bosons. Let us discuss for each case the dominant decay modes:

Case A. After the spontaneous breaking of the gauge symmetries the nonrenormalizable part of the Lagrangian has two parts: $\mathcal{L}_{A}^{\mathrm{NR}}=\mathcal{L}_{A 1}^{\mathrm{NR}}+\mathcal{L}_{A 2}^{\mathrm{NR}}$. The first one reads:

$$
\mathcal{L}_{\mathrm{A} 1}^{\mathrm{NR}}=\frac{1}{\Lambda^{2}}\left(\frac{-i g_{\phi}}{4} A_{\mu}^{3}\left(\eta^{\prime} \eta^{\prime}+2 \eta^{\prime} v_{\phi}+v_{\phi}^{2}\right) \frac{1}{2}\left(h^{\prime} \partial_{\mu} h^{\prime}+v \partial_{\mu} h^{\prime}\right)\right)+\text { h.c. },
$$

which induces the decay of the dark matter particle into scalars, by means of Eqs. (2.3): $A \rightarrow \eta \eta, A \rightarrow h \eta$ and $A \rightarrow h h$. The corresponding decay rates are:

$$
\begin{aligned}
\Gamma(A \rightarrow \eta \eta) & =\frac{1}{3} \frac{1}{16 \pi} \frac{g_{\phi}^{2}}{256 \Lambda^{4}}\left(\sin ^{2} \beta v_{\phi}^{2}+v_{\phi} v \sin 2 \beta\right)^{2} \frac{\sqrt{\left(M_{A}^{2}-4 M_{\eta}^{2}\right)^{3}}}{M_{A}^{2}}, \\
\Gamma(A \rightarrow h \eta) & =\frac{1}{3} \frac{1}{64 \pi} \frac{g_{\phi}^{2}}{256 \Lambda^{4}}\left(v_{\phi}^{2} \sin 2 \beta+4 v v_{\phi} \cos ^{2} \beta\right)^{2} \frac{\sqrt{\lambda\left(M_{A}, M_{h}, M_{\eta}\right)^{3}}}{M_{A}^{5}} \\
\Gamma(A \rightarrow h h) & =\frac{1}{3} \frac{1}{16 \pi} \frac{g_{\phi}^{2}}{256 \Lambda^{4}}\left(\cos ^{2} \beta v_{\phi}^{2}-v_{\phi} v \sin 2 \beta\right)^{2} \frac{\sqrt{\left(M_{A}^{2}-4 M_{h}^{2}\right)^{3}}}{M_{A}^{2}} .
\end{aligned}
$$

with $\lambda\left(M_{A}, m_{1}, m_{2}\right)=M_{A}^{4}+m_{2}^{4}+m_{1}^{4}-2\left(m_{1}^{2}+m_{2}^{2}\right) M_{A}^{2}-2 m_{1}^{2} m_{2}^{2}$.

In addition, there is a second term in the non-renormalizable Lagrangian:

$$
\mathcal{L}_{\mathrm{A} 2}^{\mathrm{NR}}=\frac{1}{\Lambda^{2}}\left(\frac{-i g_{\phi}}{4} A_{\mu}^{3}\left(\eta^{\prime} \eta^{\prime}+2 \eta^{\prime} v_{\phi}+v_{\phi}^{2}\right) \frac{i e}{4} B_{\mu}\left(h^{\prime} h^{\prime}+2 v h^{\prime}+v^{2}\right)\right)+\text { h.c. },
$$

which induces decays into a gauge boson and the hidden sector and standard model Higgs bosons, $A \rightarrow \gamma \eta, A \rightarrow Z \eta, A \rightarrow \gamma h$ and $A \rightarrow Z h$, with rates:

$$
\begin{aligned}
\Gamma(A \rightarrow \gamma \eta)= & \frac{1}{3} \frac{1}{16 \pi} \frac{3 e^{2} g_{\phi}^{2} \cos ^{2} \theta_{W}\left(\cos \beta v_{\phi} v^{2}+\sin \beta v v_{\phi}^{2}\right)^{2}}{64 \Lambda^{4}} \frac{\left(M_{A}^{2}-M_{\eta}^{2}\right)}{M_{A}^{3}} \\
\Gamma(A \rightarrow Z \eta)= & \frac{1}{3} \frac{1}{64 \pi} \frac{e^{2} g_{\phi}^{2} \sin ^{2} \theta_{W}\left(\cos \beta v_{\phi} v^{2}+\sin \beta v v_{\phi}^{2}\right)^{2}}{64 \Lambda^{4}} \times \\
& \times\left(8+\frac{\left(M_{A}^{2}-M_{\eta}^{2}+M_{Z}^{2}\right)^{2}}{M_{A}^{2} M_{Z}^{2}}\right) \frac{\sqrt{\lambda\left(M_{A}, M_{Z}, M_{\eta}\right)}}{M_{A}^{3}}
\end{aligned}
$$

The decay rates into $\gamma h$ and into $Z h$ are obtained using Eqs. (2.3) and $M_{\eta} \rightarrow M_{h}$.

Case B. At low energies the non-renormalizable Lagrangian for case $\mathrm{B}$ is $\mathcal{L}_{B}^{\mathrm{NR}}=\mathcal{L}_{A 1}^{\mathrm{NR}}-$ $\mathcal{L}_{A 2}^{\mathrm{NR}}$, where $\mathcal{L}_{A 1}^{\mathrm{NR}}$ and $\mathcal{L}_{A 2}^{\mathrm{NR}}$ are given in Eqs. (2.8) and 2.10) (note the change of sign in the operator involving two gauge bosons compared to the case A). Thus, the decay modes and rates are identical to the case $\mathrm{A}$. 
Case C. After the symmetry breaking the Lagrangian for this dimension six operator reads as

$$
\mathcal{L}_{C}^{\mathrm{NR}}=\frac{1}{\Lambda^{2}} 2 A_{\nu}^{3}\left(\partial_{\mu} B_{\nu}-\partial_{\nu} B_{\mu}\right) \partial_{\mu} \eta^{\prime}\left(\eta^{\prime}+v_{\phi}\right)+\text { h.c. } .
$$

Remarkably it leads only to the following two body decays

$$
\begin{aligned}
\Gamma(A \rightarrow \gamma \eta)= & \frac{1}{3} \frac{1}{16 \pi} \frac{g_{\phi}^{2} v_{\phi}^{2} \cos ^{2} \beta \cos ^{2} \theta_{W}}{8 \Lambda^{4} M_{A}^{3}}\left(\left(M_{A}^{2}-M_{\eta}^{2}\right)^{3}+\frac{M_{\eta}^{2}}{M_{A}^{2}}\left(M_{A}^{4}+M_{\eta}^{4}\right)\left(M_{A}^{2}-M_{\eta}^{2}\right)\right) \\
\Gamma(A \rightarrow Z \eta)= & \frac{1}{3} \frac{1}{16 \pi} \frac{g_{\phi}^{2} v_{\phi}^{2} \cos ^{2} \beta \sin ^{2} \theta_{W}}{16 \Lambda^{4} M_{A}^{3}} \sqrt{\lambda\left(M_{A}, M_{Z}, M_{\eta}\right)} \times \\
& \times\left(2\left(M_{A}^{2}-M_{\eta}^{2}\right)^{2}-3 M_{Z}^{2}\left(M_{A}^{2}-2 M_{\eta}^{2}\right)-\frac{M_{Z}^{4}\left(M_{Z}-M_{\eta}\right)^{2}}{M_{A}^{2}}\right) .
\end{aligned}
$$

together with the corresponding decay channels into $\gamma h$ and $Z h$, which can be derived using Eqs. 2.3) and substituting $M_{\eta} \rightarrow M_{h}$.

Case D. The non-renormalizable part of the Lagrangian contains two terms: $\mathcal{L}_{D}^{\mathrm{NR}}=$ $\mathcal{L}_{D_{1}}^{\mathrm{NR}}+\mathcal{L}_{D_{2}}^{\mathrm{NR}}$. It contains a kinetic mixing term:

$$
\mathcal{L}_{D_{1}}^{\mathrm{NR}}=\frac{v_{\phi}^{2}}{2 \Lambda^{2}} F_{\mu \nu}^{3} F^{\mu \nu Y},
$$

which leads to decays into fermion pairs, into $W^{+} W^{-}$, into $Z \eta$ and into $Z h$.

For the two-body decay into fermion pairs we obtain (neglecting fermion masses)

$$
\Gamma(A \rightarrow f \bar{f})=\frac{1}{3} \frac{C}{64 \pi} \frac{g^{2} v_{\phi}^{4}}{\Lambda^{4}}\left(\left(d_{V}^{f}\right)^{2}+\left(d_{A}^{f}\right)^{2}\right) M_{A},
$$

where we have introduced a color factor which is $C=1$ for leptons and $C=3$ for quarks. The effective vector and axial couplings to neutrinos, charged leptons, up-type and down-type quarks are given by

$$
\begin{aligned}
& d_{V}^{\nu}=d_{A}^{\nu}=-d_{A}^{e}=d_{A}^{u}=-d_{A}^{d}=-\frac{1}{2} \frac{M_{A}^{2}}{M_{A}^{2}-M_{Z}^{2}}, \\
& d_{V}^{e}=\left(2 \sin ^{2} \theta_{W}-\frac{1}{2}\right) \frac{M_{Z}^{2}}{M_{Z}^{2}-M_{A}^{2}}-\frac{3}{2}, \\
& d_{V}^{u}=\left(\frac{1}{2}-\frac{4}{3} \sin ^{2} \theta_{W}\right) \frac{M_{Z}^{2}}{M_{Z}^{2}-M_{A}^{2}}+\frac{5}{6}, \\
& d_{V}^{d}=\left(\frac{2}{3} \sin ^{2} \theta_{W}-\frac{1}{2}\right) \frac{M_{Z}^{2}}{M_{Z}^{2}-M_{A}^{2}}-\frac{1}{6} .
\end{aligned}
$$

The other decay widths are given by

$$
\begin{aligned}
\Gamma\left(A \rightarrow W^{+} W^{-}\right)= & \frac{v_{\phi}^{4}}{16 \Lambda^{4}} \frac{\alpha \cos ^{2} \theta_{W}}{12} M_{A}\left(\frac{M_{A}}{M_{W}}\right)^{4}\left(\frac{M_{Z}^{2}}{M_{Z}^{2}-M_{A}^{2}}\right)^{2} \times \\
& \times\left(1+20 \frac{M_{W}^{2}}{M_{A}^{2}}+12 \frac{M_{W}^{4}}{M_{A}^{4}}\right)\left(1-\frac{4 M_{W}^{2}}{M_{A}^{2}}\right)^{3 / 2}
\end{aligned}
$$


and

$$
\begin{aligned}
\Gamma(A \rightarrow Z \eta)= & \frac{1}{3} \frac{1}{256 \pi} \frac{g^{2} M_{Z}^{2} \sin ^{2} \beta}{\cos ^{2} \theta_{W}} \frac{v_{\phi}^{4}}{\Lambda^{4} M_{A}}\left(\frac{M_{Z}^{2}}{M_{Z}^{2}-M_{A}^{2}}-\sin \theta_{W}\right)^{2} \times \\
& \times\left(10+\frac{M_{A}^{2}}{M_{Z}^{2}}+\frac{M_{Z}^{2}}{M_{A}^{2}}+\frac{M_{\eta}^{2}\left(M_{\eta}^{2}-2 M_{A}^{2}-2 M_{Z}^{2}\right)}{M_{A}^{2} M_{Z}^{2}}\right) \times \\
& \times \sqrt{\left(1-\left(M_{Z}+M_{\eta}\right)^{2} / M_{A}^{2}\right)\left(1-\left(M_{Z}-M_{\eta}\right)^{2} / M_{A}^{2}\right)} .
\end{aligned}
$$

For the corresponding decay channels into $Z h$, the last equation holds with the replacement of the physical $h$ boson from Eqs. 2.3.

The second term, on the other hand, reads:

$$
\mathcal{L}_{D_{2}}^{\mathrm{NR}}=\frac{1}{\Lambda^{2}} v_{\phi} \partial_{\mu} A_{\nu}^{3}\left(\partial_{\mu} B_{\nu}-\partial_{\nu} B_{\mu}\right) \eta^{\prime},
$$

which leads to two body decays involving the hidden sector Higgs $\eta$, using Eqs. 2.3, and a gauge boson, with rates:

$$
\begin{aligned}
\Gamma(A \rightarrow \gamma \eta)= & \frac{1}{3} \frac{1}{32 \pi} \frac{v_{\phi}^{2}}{\Lambda^{4}} \cos ^{2} \theta_{W} \cos ^{2} \beta \frac{\left(M_{A}^{2}-M_{\eta}^{2}\right)^{3}}{M_{A}^{3}} \\
\Gamma(A \rightarrow Z \eta)= & \frac{1}{3} \frac{1}{32 \pi} \frac{v_{\phi}^{2}}{\Lambda^{4}} \sin ^{2} \theta_{W} \cos ^{2} \beta \times \\
& \times\left(\left(M_{A}^{2}-M_{\eta}^{2}+M_{Z}^{2}\right)^{2}+2 M_{Z}^{2} M_{A}^{2}\right) \frac{\sqrt{\lambda\left(M_{A}, M_{Z}, M_{\eta}\right)}}{M_{A}^{3}} .
\end{aligned}
$$

The decay into $Z h$ and $\gamma h$ can be obtained with the substitution $\cos \beta \rightarrow \sin \beta$.

If $\beta \neq 0$ and $\beta \neq \pi / 2$, then we have interference between the decay coming from $\mathcal{L}_{D_{1}}^{N R}$ and $\mathcal{L}_{D_{2}}^{N R}$, which lead to the corrections:

$$
\begin{aligned}
\delta \Gamma(A \rightarrow Z \eta)= & -\frac{1}{3} \frac{3}{64 \pi} \frac{v_{\phi}^{3}}{\Lambda^{4}} \frac{g \sin \theta_{W} M_{Z} \sin 2 \beta}{\cos \theta_{W}}\left(\frac{M_{Z}^{2}}{M_{Z}^{2}-M_{A}^{2}}-\sin \theta_{W}\right) \times \\
& \times\left(M_{A}^{2}-M_{\eta}^{2}+M_{Z}^{2}\right) \frac{\sqrt{\lambda\left(M_{A}, M_{Z}, M_{\eta}\right)}}{M_{A}^{3}}, \\
\delta \Gamma(A \rightarrow Z h)= & \frac{1}{3} \frac{3}{64 \pi} \frac{v_{\phi}^{3}}{\Lambda^{4}} \frac{g \sin \theta_{W} M_{Z} \sin 2 \beta}{\cos \theta_{W}}\left(\frac{M_{Z}^{2}}{M_{Z}^{2}-M_{A}^{2}}-\sin \theta_{W}\right) \times \\
& \times\left(M_{A}^{2}-M_{h}^{2}+M_{Z}^{2}\right) \frac{\sqrt{\lambda\left(M_{A}, M_{Z}, M_{h}\right)}}{M_{A}^{3}} .
\end{aligned}
$$

\section{Cosmic ray signatures of hidden vector dark mat- ter decay}

In this section we firstly give a short introduction to the propagation of gamma rays and charged cosmic rays through the Galaxy, and secondly discuss the typical cosmic-ray 
signatures of hidden vector dark matter, including strong gamma-ray lines and possible contributions to the anti-matter fluxes.

\subsection{Cosmic ray propagation}

The hidden gauge boson decay produces a high energy flux of stable particles, such as gamma rays, electrons, positrons, antiprotons, neutrinos and antideuterons. The flux of high-energy cosmic rays depends essentially on the scale of custodial symmetry breaking, which is thus constrained by the requirement that the predicted fluxes do not exceed the observed fluxes. We will show that a typical signature of the hidden vector dark matter model is a prominent gamma-ray line, with values of the custodial symmetry breaking scale close to the Grand Unification scale and in reach of the Fermi LAT dark matter searches. Furthermore, as mentioned in the introduction, experiments measuring the positron fraction and the total electron plus positron flux indicate the existence of an additional source of electrons and positrons at high energies, but no additional source of antiprotons. We will also explore the possibility that the decay of dark matter hidden gauge bosons could be the origin of such excesses.

The production rate of particle $i=e, \gamma, \bar{p}$ per unit energy and unit volume at a position $\vec{r}$ with respect to the center of the Milky Way is given by

$$
Q_{i}(E, \vec{r})=\frac{\rho(\vec{r})}{M_{\mathrm{DM}} \tau_{\mathrm{DM}}} \frac{d N_{i}}{d E},
$$

where $d N_{i} / d E$ is the corresponding energy spectrum of particle $i$ produced in the decay, and $\rho(\vec{r})$ is the density profile of dark matter particles in the Milky Way halo. We will adopt in this paper the spherically symmetric NFW density profile [42] for definiteness,

$$
\rho(r) \propto \frac{1}{\left(r / r_{c}\right)\left[1+\left(r / r_{c}\right)\right]^{2}},
$$

normalized to $0.3 \mathrm{GeV} / \mathrm{cm}^{3}$ at the position of the sun, $r=8.5 \mathrm{kpc}$, although our results do not depend much on the specific form of the halo profile.

Gamma-rays, contrary to electrons, positrons and antiprotons, which will be discussed below, do not diffuse and carry information about their spatial origin. The gammaray signal from dark matter decay consists of several components. The most important one is related to the prompt radiation (e.g. final state radiation) produced in the decay of DM particles inside the Milky Way halo. It depends on the halo density profile, and although the halo profile is expected to be approximately isotropic, the corresponding flux at Earth exhibits a dipole-like anisotropy which is due to the off-set between the Sun 
and the Galactic center and which can be as large as 20-30\% for dark matter lifetimes of the order of $10^{26} \mathrm{~s}$ [43]. In contrast, the extragalactic prompt component of the $\gamma$-ray signal, which stems from the decay of dark matter particles at cosmological distances, is isotropic. At energies around $10 \mathrm{GeV}$ or below, the magnitude of the halo and extragalactic fluxes are comparable when looking in direction of the anti-galactic center, whereas at higher energies around and above $100 \mathrm{GeV}$ the inelastic scattering between $\gamma$-rays and the intergalactic background light reduces the extragalactic component considerably (see Ref. 43] for a discussion). In the plots we assume a $10 \%$ energy resolution, and we show also the H.E.S.S [13, 14] results for the electron + positron (+ gamma) flux in the TeV region, which acts like an upper bound on the isotropic flux.

For details about the adopted calculation of the electron, anti-matter and gamma-ray fluxes we refer to Refs. [16, 43].

Electrons and positrons from dark matter decay loose their energy mainly via interaction with the Galactic magnetic field and the interstellar radiation field (ISRF). In the first case (assuming injection energies of the order of $1 \mathrm{TeV}$ ) synchrotron radiation in the radio band with frequencies $\mathcal{O}(0.1-100 \mathrm{GHz})$ is produced and potentially observable (see e.g. Refs. [44, 45, 46]). In the second case, the inverse Compton scattering (ICS) of electrons and positrons on the ISRF (which includes the cosmic microwave background, thermal dust radiation and starlight) produces a second component of gamma rays with energies between $100 \mathrm{MeV}$ and $1 \mathrm{TeV}$ [47, 48]. However, since electron and positron fluxes are always relatively weak in the decay channels we consider we will neglect ICS radiation throughout this work for simplicity.

After being produced in the decay of dark matter particles, electrons and positrons scatter on irregularities of the Galactic magnetic field before reaching the Earth, which results in a wash-out of directional information. Their propagation is commonly described by a diffusion model, whose free parameters are tuned to reproduce the observed cosmicray nuclei fluxes [49]. As propagation parameters we will adopt the ones of the MED propagation model defined in [50], which provide the best fit to the Boron-to-Carbon (B/C) ratio: $\delta=0.70, K_{0}=0.0112 \mathrm{kpc}^{2} / \mathrm{Myr}$ and $L=4 \mathrm{kpc}$. Our conclusions, however, are rather insensitive to the choice of propagation parameters. The astrophysical background in the $e^{ \pm}$-channel is mainly due to primary electrons, which are presumably produced in supernova remnants, and due to secondary positrons, produced in the interaction of cosmic-rays with the galactic gas. For these background fluxes we adopt the "Model 0" presented by the Fermi collaboration in [12], which fits well the low-energy data points of the total electron plus positron and the positron fraction. We allow, however, for a $10 \%$ rescaling of the electron background in order to improve the agreement 
of the total flux to the data.

The antiproton propagation in the Galaxy is analogous to the propagation of electrons and positrons. However, since antiprotons are much heavier than electrons and positrons, energy losses are negligible. Furthermore, antiproton propagation is affected by convection, which accounts for the drift of antiprotons away from the disk induced by the Milky Way's Galactic wind. For predictions of the antiproton flux we show an error band, corresponding to the MIN and MAX model of Ref. [50]. In our plots we present actually the $\bar{p} / p$-ratio, where we adopt the proton and anti-proton backgrounds from Ref. [51].

For both, electrons/positrons and anti-protons, the fluxes at the top of the atmosphere can differ considerably from the interstellar fluxes at energies smaller than $\sim 10$ $\mathrm{GeV}$, due to solar modulation effects. To take this effect into account, we adopt the force field approximation [52] with $\phi_{F}=550 \mathrm{MV}$ [53].

The main background in the $\gamma$-ray channel is the diffuse emission of our Galaxy, which is mainly due to interactions of cosmic rays with the galactic gas and the ISRF [54]. This component is by far strongest in the galactic disk region, and it turns out that exotic fluxes from dark matter decay would dominantly show up at higher latitudes, away from the disk. For this reason they could be misidentified as contribution to the extragalactic gamma-ray background (although they can be distinguished by their large scale anisotropy, see Ref. [43]). In this work we will show predictions for the averaged gamma-ray flux in the region $0 \leq l \leq 360^{\circ}, 10^{\circ} \leq|b| \leq 90^{\circ}$, which offers the best strategy for searching gamma-ray lines from dark matter decay [55].

\subsection{Gamma-ray lines}

The existence of two-body decay modes with gamma-ray lines in the final state are a generic prediction of hidden vector dark matter models. We will discuss this for each possible operator separately, Eqs. 2.4 - 2.7.

Case A and B. In cases A and B, Eqs. (2.4) and (2.5), the dark matter particle decays either into two scalar particles $(\eta \eta, h \eta, h h)$ or into a gauge boson and a scalar particle $(\gamma \eta, \gamma h, Z \eta, Z h)$. Whether the dark matter particle decays preferentially into two scalar particles or into a gauge boson and a scalar particle depends on the details of the model. In both cases, the fragmentation and decay of the Higgs boson or the hidden sector $\eta$ boson could produce a sizable flux of electrons, positrons and antiprotons. Unfortunately, the electrons and positrons produced in fragmentations cannot explain the PAMELA and 


\begin{tabular}{c|cccccc}
\hline \hline Benchmark & $M_{A}$ & $g_{\phi}$ & $v_{\phi}$ & $M_{\eta}$ & $M_{h}$ & $\sin \beta$ \\
\hline \hline 1 & $300 \mathrm{GeV}$ & 0.55 & $1090 \mathrm{GeV}$ & $30 \mathrm{GeV}$ & $150 \mathrm{GeV}$ & $\approx 0$ \\
2 & $600 \mathrm{GeV}$ & 0.6 & $2000 \mathrm{GeV}$ & $30 \mathrm{GeV}$ & $120 \mathrm{GeV}$ & $\approx 0$ \\
3 & $14 \mathrm{TeV}$ & 12 & $2333 \mathrm{GeV}$ & $500 \mathrm{GeV}$ & $145 \mathrm{GeV}$ & $\approx 0$ \\
4 & $1550 \mathrm{GeV}$ & 2.1 & $1457 \mathrm{GeV}$ & $1245 \mathrm{GeV}$ & $153 \mathrm{GeV}$ & 0.25 \\
\hline \hline
\end{tabular}

Table 1: Benchmark points used for the calculation of cosmic-ray signatures.

Fermi excesses and moreover the PAMELA measurements on the antiproton-to-proton ratio set very stringent constraints on possible new sources of antiprotons. Interestingly, even if the scale $\Lambda$ is increased in order to avoid an antiproton excess, the generically present gamma-ray lines can still be intense enough to be observed in experiments, due to the enormous sensitivity of dark matter line searches.

This is illustrated in Figs. 1 and 2, where we show the predictions for the positron fraction, total electron plus positron flux, antiproton-to-proton fraction and gammaray flux for two generic scenarios, namely the benchmark points 1 and 2 defined in Tab. 1. These choices of parameters can successfully reproduce the observed dark matter abundance and are consistent with all present laboratory constraints. We also show in the plots for the positron fraction the results from PAMELA [9], HEAT [56], CAPRICE [57] and AMS-01 [58]; for the total electron plus positron flux, the results from Fermi [11], H.E.S.S. [13, 14], BETS, PPB-BETS [59], ATIC [60], HEAT, CAPRICE and AMS01; for the antiproton-to-proton ratio, the results from PAMELA [17], BESS95 [61, BESS95/97 [62], CAPRICE94 [63], CAPRICE98 [64] and IMAX [65] and for the gammaray flux, the preliminary data from the Fermi-LAT in the region between $10^{\circ}$ and $90^{\circ}$, as well as the extraction of the extragalactic flux from these data [66]. In the gamma-ray plot, we also show the H.E.S.S. results for the electron + positron (+gamma) flux at high energies, which yields also an upper bound on the overall isotropic gamma-ray flux.

The branching ratios for these two benchmark points are listed in Tab. 2. Benchmark point 1 is characterized by large branching ratios into gauge boson and Higgs, being the decay into a monoenergetic gamma line the dominant channel. On the other hand, since kinematically allowed, benchmark point 2 is characterized by a large branching ratio into two Higgs bosons. It is interesting that, even though the decay mode into monoenergetic gamma rays is subdominant in this benchmark point, the gamma-ray line still is a very prominent feature in the gamma-ray energy spectrum.

We estimate that, in the limit $v_{\phi} \gg v, \beta \rightarrow 0$, the decay rate into $\gamma h$ is given by:

$$
\Gamma(A \rightarrow \gamma h)^{-1}=1.5 \times 10^{28} \mathrm{~s}\left(\frac{\Lambda}{2 \times 10^{15} \mathrm{GeV}}\right)^{4}\left(\frac{1 \mathrm{TeV}}{v_{\phi}}\right)^{2}\left(\frac{100 \mathrm{GeV}}{M_{A}}\right)
$$




\begin{tabular}{c|ccccccc}
\hline \hline Benchmark & $\eta \eta$ & $h \eta$ & $h h$ & $\gamma \eta$ & $Z \eta$ & $\gamma h$ & $Z h$ \\
\hline \hline 1 & - & 0.09 & - & 0.04 & 0.02 & 0.65 & 0.20 \\
2 & - & 0.04 & 0.62 & 0.002 & 0.003 & 0.15 & 0.18 \\
3 & - & 0.04 & 0.80 & $3 \times 10^{-6}$ & 0.002 & 0.0003 & 0.16 \\
\hline \hline
\end{tabular}

Table 2: Branching Ratios for Case A.

The Fermi-LAT observations of the region $|b|>10^{\circ}$ plus a $20^{\circ} \times 20^{\circ}$ square around the Galactic center constrain the dark matter lifetime to be longer than a few times $10^{28} \mathrm{~s}$ at energies below $200 \mathrm{GeV}$ [67], which is taken into account in the results shown for benchmark point 1 (see Fig. 1), where the line is around $110 \mathrm{GeV}$. Thus present experiments can probe values of the scale of custodial symmetry breaking close to the Grand Unification scale. In case of benchmark point 2 the line occurs at an energy scale above the ones probed by Fermi, so that smaller lifetime are allowed experimentally. We show results for a lifetime $1.1 \times 10^{27} \mathrm{~s}$, where the contributions to the diffuse gamma-rays around $10 \mathrm{GeV}$ and the anti-proton fluxes can be sizeable. The gamma line in this case is huge and should be seen by any experiment sensitive to these energies.

Case C. This operator, see Eq. (2.6), predicts decays only into a gauge boson and a scalar particle, either $h$ or $\eta$. A large decay branching ratio into monoenergetic gammarays is predicted unavoidably, as illustrated in Tab. 3 for the four different benchmark scenarios. In the limit $M_{\eta} \ll M_{A}$, the decay rate into $\gamma \eta$ is given by

$$
\Gamma(A \rightarrow \gamma \eta)^{-1}=2.7 \times 10^{28} \mathrm{~s}\left(\frac{\Lambda}{4 \times 10^{15} \mathrm{GeV}}\right)^{4}\left(\frac{300 \mathrm{GeV}}{M_{A}}\right)^{5}
$$

which can make the gamma-ray line observable at the Fermi-LAT for values of the scale of custodial symmetry close to the Grand Unification Scale, especially for large dark matter masses. The cosmic ray signatures of benchmark point 1 for case $\mathrm{C}$ are very similar to case A, cf. Fig. 1. On the other hand, we show in Fig. 3 the predictions for benchmark point 3 with a very large dark matter mass of $14 \mathrm{TeV}$, which predicts a strong line at very high energies and only small contributions to positrons and anti-protons.

One feature of the model that is in principle present for each operator, and which we want to illustrate for case $\mathrm{C}$, is the general existence of two independent gamma-ray lines. These lines stem from the decay into $\gamma h$ and $\gamma \eta$ and would appear at different energies as long as the higgs and the $\eta$ masses are not too degenerate. In case $\mathrm{C}$ both of the decay channels are in general open as long as $\sin \beta \neq 0$, which is the case for benchmark point 4 in Tab. 1. In Fig. 4 we show the corresponding cosmic-ray fluxes. Most interestingly the gamma-ray flux exhibits two strong peaks in this case, located at 270 and $770 \mathrm{GeV}$. 

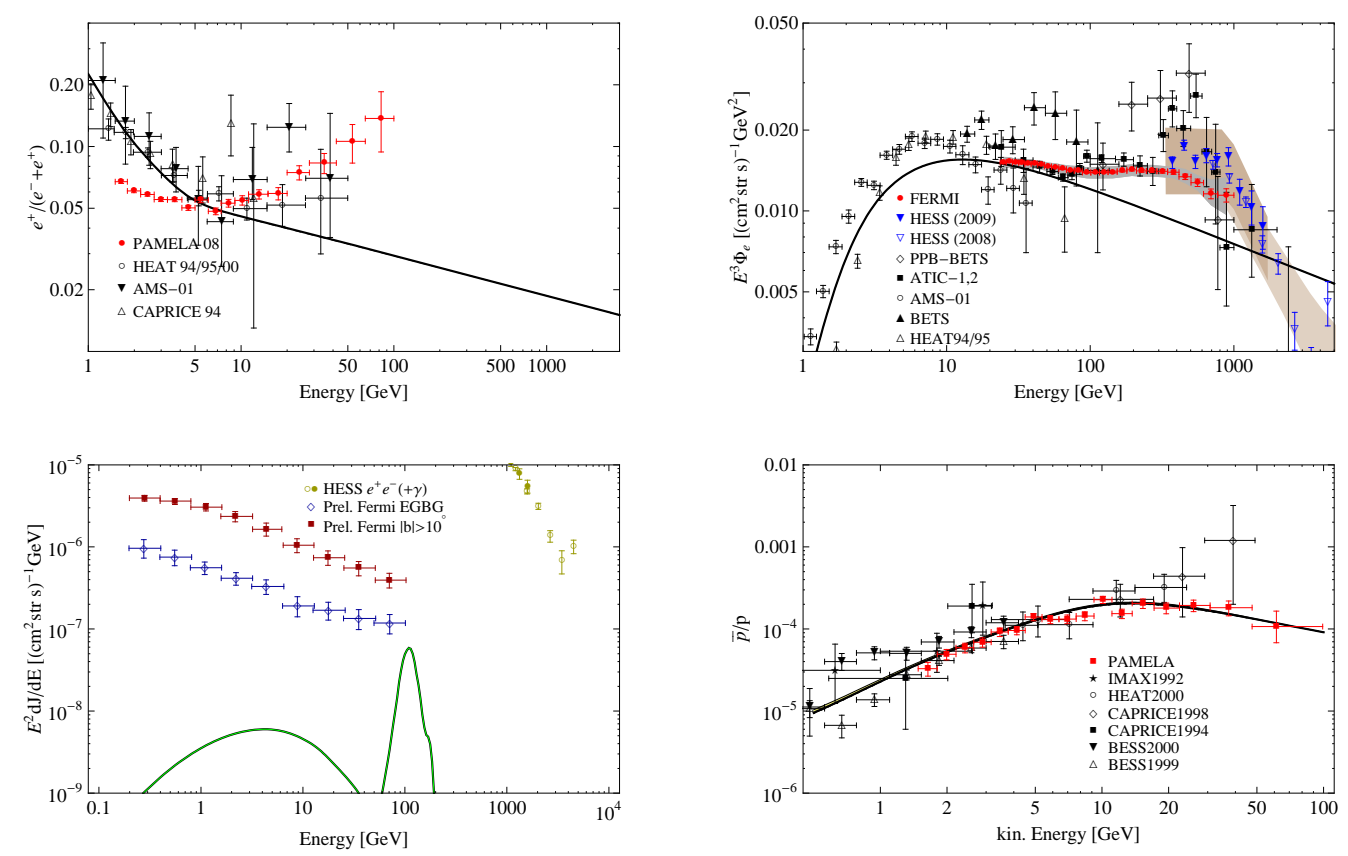

Figure 1: Predictions for case A, benchmark 1, with $\tau_{\mathrm{DM}}=1.7 \times 10^{28} \mathrm{~s}(\Lambda=2.9 \times$ $10^{15} \mathrm{GeV}$ ). The upper panels show the positron fraction (left) and the total electron + positron flux (right) compared with experimental data. Dashed lines show the adopted astrophysical background, solid lines are background + dark matter signal (which overlap the background in this plot). The lower left panel shows the gamma-ray signal from dark matter decay, whereas the lower right panel shows the $\bar{p} / p$-ratio: background (dashed line) and overall flux (solid lines, again identical with background).

Case D. This operator, see Eq. (2.7), is particularly interesting since it induces a kinetic mixing between the $U(1)_{Y}$ of hypercharge and one of the hidden $S U(2)$ gauge bosons. As a result two-body decay modes into lepton and quark pairs are allowed, in contrast to the other operators. This leads to interesting implications for the electron/positron flux that will be discussed shortly below.

Here we firstly emphasize that again the operator also predicts two-body decay into $\gamma h$, which could be observable in different parts of the parameter space. The inverse decay rate reads, for $M_{\eta} \ll M_{A}$ :

$$
\Gamma(A \rightarrow \gamma \eta)^{-1}=2.4 \times 10^{28} \mathrm{~s}\left(\frac{\Lambda}{7 \times 10^{15} \mathrm{GeV}}\right)^{4}\left(\frac{1 \mathrm{TeV}}{v_{\phi}}\right)^{2}\left(\frac{300 \mathrm{GeV}}{M_{A}}\right)^{3}
$$

and shows that the line could be observed by Fermi LAT for scales of the custodial symmetry breaking close to the Grand Unification scale. For these large lifetimes around $10^{28} \mathrm{~s}$ contributions to the anti-matter channel would be negligible. However, if the line lies above around $300 \mathrm{GeV}$ and out of reach of Fermi LAT, shorter lifetimes cannot be 

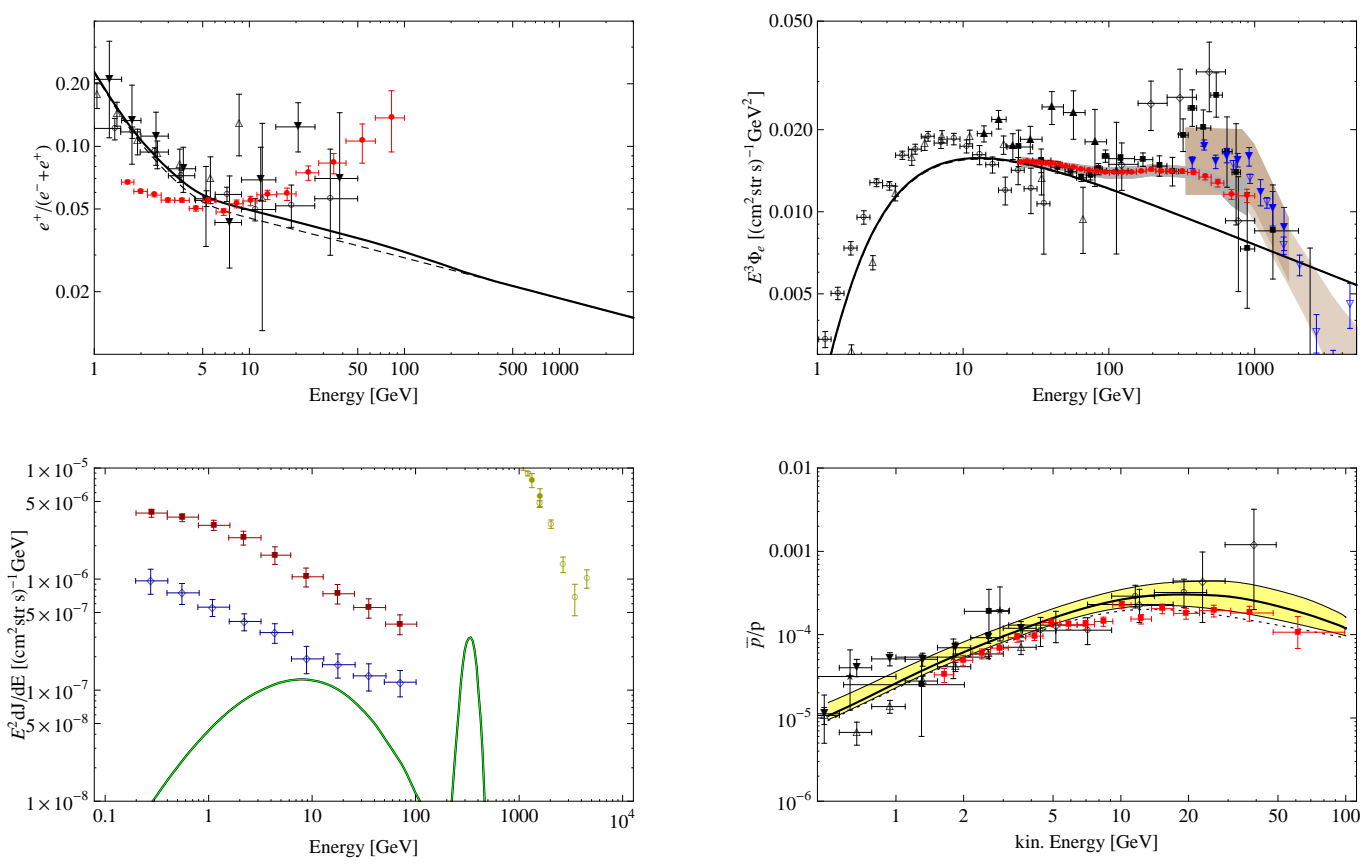

Figure 2: Like Fig. 1, but for case A, benchmark 2, with $\tau_{\mathrm{DM}}=1.1 \times 10^{27} \mathrm{~s}(\Lambda=$ $\left.3.7 \times 10^{15} \mathrm{GeV}\right)$. The yellow band shows the uncertainty in the anti-proton flux due to the propagation model parameters.

excluded and the anti-matter fluxes can be sizeable.

\subsection{Positron flux}

Here we will briefly discuss the predictions for the anti-matter fluxes concentrating on case D, since this operator features two-body decay into fermions pairs due to effective kinetic mixing between hidden sector and the hypercharge $U(1)_{Y}$. The corresponding branching ratios are listed in Tab. 4. In the cases with lower dark matter mass, the branching ratio into hard leptons (and in particular electrons) is sizable. Namely, in the limit $M_{A} \gg M_{Z}$ the inverse decay rate into charged lepton pairs is given by

$$
\Gamma\left(A \rightarrow \ell^{+} \ell^{-}\right)^{-1}=2.6 \times 10^{27} \mathrm{~s}\left(\frac{\Lambda}{7 \times 10^{15} \mathrm{GeV}}\right)^{4}\left(\frac{1 \mathrm{TeV}}{v_{\phi}}\right)^{4}\left(\frac{300 \mathrm{GeV}}{M_{A}}\right)
$$

which can produce a steep rise in the observed positron fraction for values of the scale of custodial symmetry breaking of the order of the Grand Unification scale.

As an example, we show in Fig. 5 the predictions for the cosmic ray fluxes for benchmark point 2 . For a scale of custodial symmetry breaking $\Lambda=7.2 \times 10^{15} \mathrm{GeV}$, which is close to the Grand Unification scale, the gamma ray spectrum shows a intense gammaray line at $300 \mathrm{GeV}$, in agreement with current observations. On the other hand, the 


\begin{tabular}{c|cccc}
\hline \hline Benchmark & $Z \eta$ & $\gamma \eta$ & $Z h$ & $\gamma h$ \\
\hline \hline 1 & 0.19 & 0.81 & 0 & 0 \\
2 & 0.22 & 0.78 & 0 & 0 \\
3 & 0.23 & 0.77 & 0 & 0 \\
4 & 0.028 & 0.79 & 0.041 & 0.14 \\
\hline \hline
\end{tabular}

Table 3: Branching Ratios for Case C, including benchmark point 4 which features decay channels with $h$ in the final state.
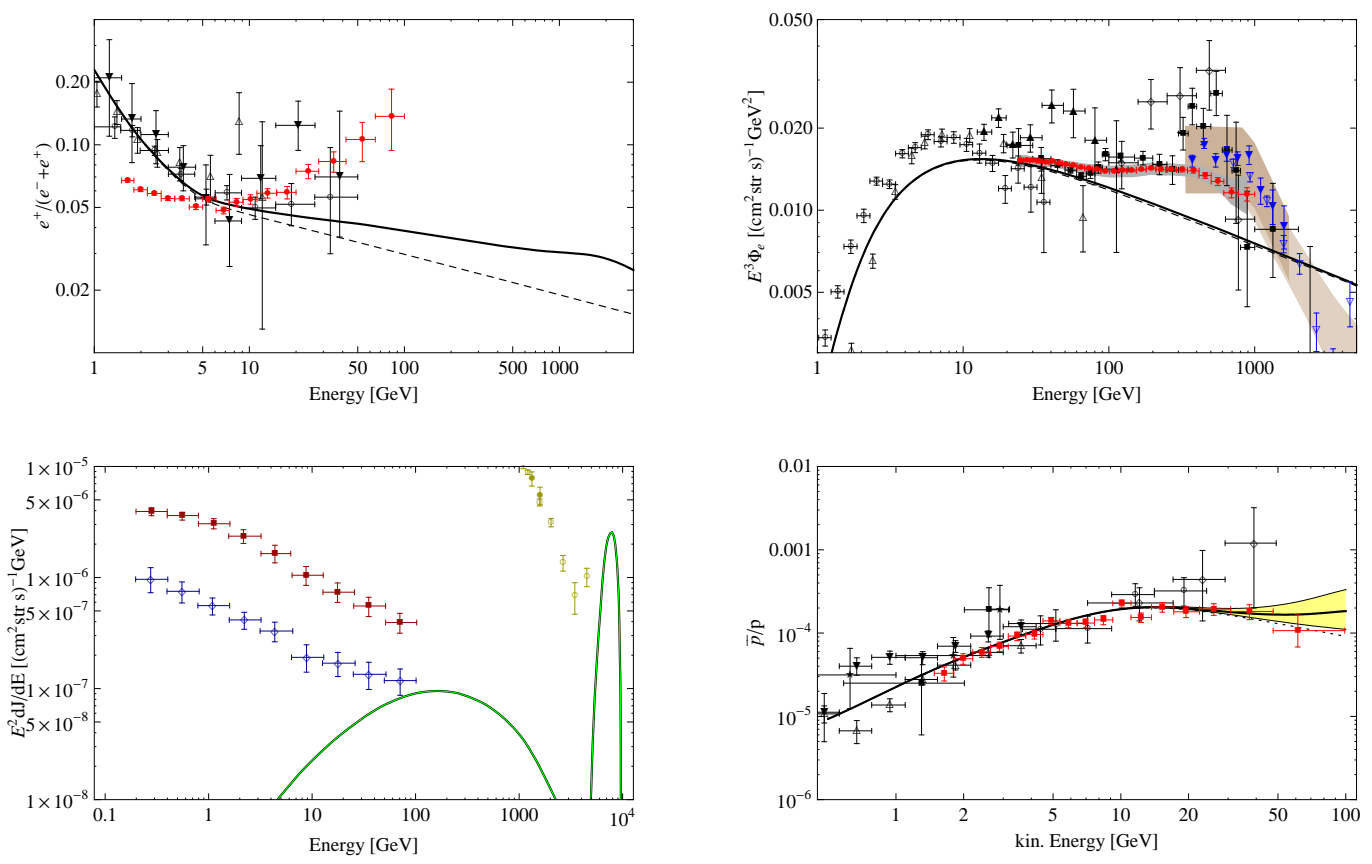

Figure 3: Like Fig. 1, but for case $\mathrm{C}$, benchmark 3, with $\tau_{\mathrm{DM}}=6.0 \times 10^{26} \mathrm{~s}(\Lambda=$ $\left.2.0 \times 10^{17} \mathrm{GeV}\right)$.

positron fraction shows a steep rise which could partially, although not totally, contribute to the PAMELA positron excess. Moreover, the decay into charged leptons is necessarily accompanied by a decay into quarks, which produce a sizable antiproton flux and is in some tension with the observations. This is a generic feature of the decay mode and hence it is unlikely that it contributes the dominant part to the observed positron excess.

In more generality we found that the PAMELA and Fermi results can be reproduced in principle by the model, but only at the price of producing a too large diffuse $\gamma$ signal, too many antiprotons (unless the dark matter is very heavy) and sometimes gamma lines above the rates allowed by the H.E.S.S. measurements in the multi $\mathrm{TeV}$ range. 

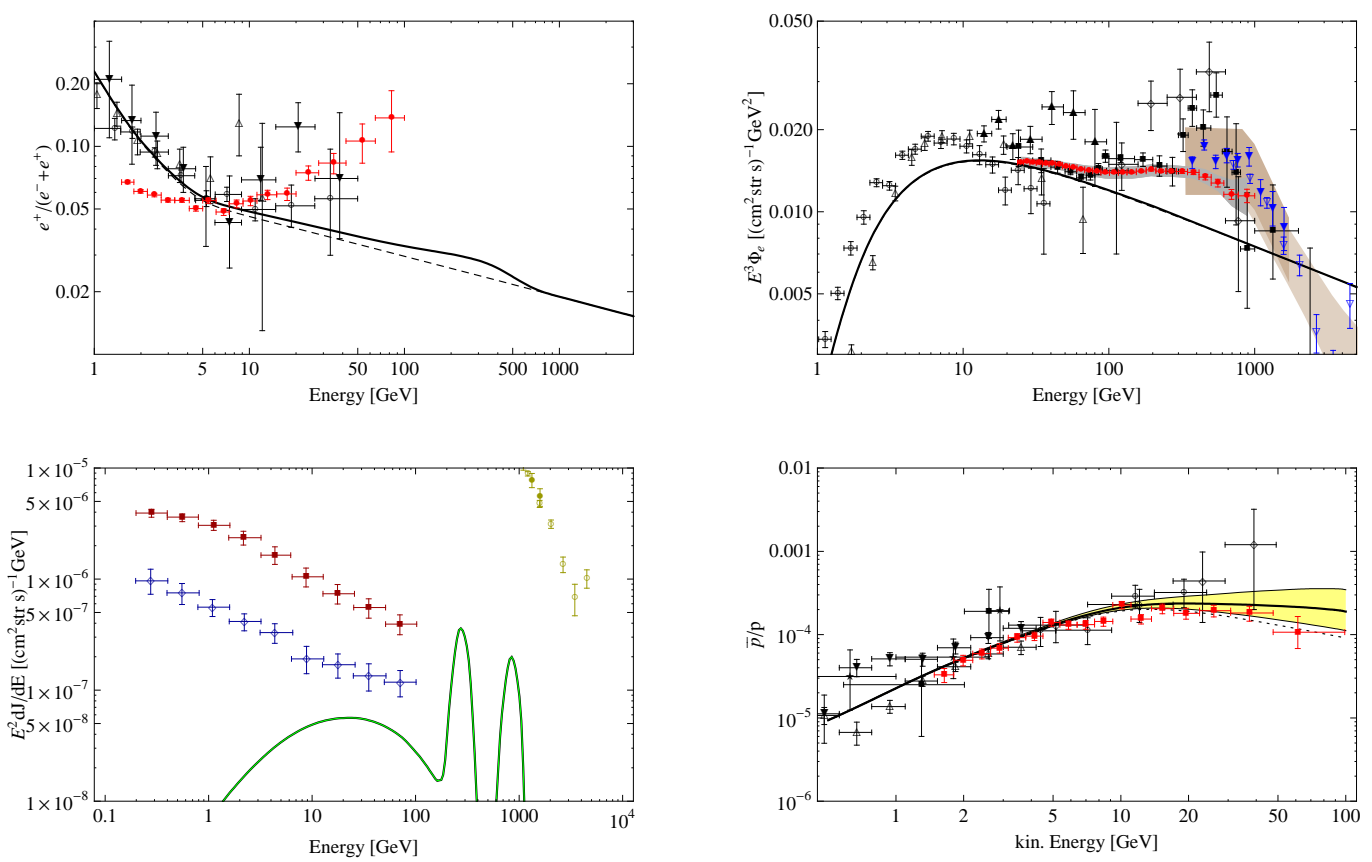

Figure 4: Like Fig. 1, but for case C, benchmark 4, with $\tau_{\mathrm{DM}}=1.6 \times 10^{27} \mathrm{~s}(\Lambda=$ $\left.1.2 \times 10^{16} \mathrm{GeV}\right)$.

\begin{tabular}{c|cccccccc}
\hline \hline Benchmark & $Z \eta$ & $Z h$ & $\gamma \eta$ & $W^{+} W^{-}$ & $\nu \bar{\nu}$ & $e^{+} e^{-}$ & $u \bar{u}$ & $d \bar{d}$ \\
\hline \hline 1 & 0.01 & 0.005 & 0.04 & 0.02 & 0.09 & 0.39 & 0.29 & 0.15 \\
2 & 0.019 & 0.004 & 0.036 & 0.014 & 0.072 & 0.35 & 0.39 & 0.12 \\
3 & 0.22 & 0.0002 & 0.73 & 0.0005 & 0.003 & 0.016 & 0.018 & 0.005 \\
\hline \hline
\end{tabular}

Table 4: Branching Ratios for Case D.

Discussion. It is intriguing that the production of a $\gamma$-ray line is a generic prediction for all possible operators that may mediate the decay of the $S U(2)_{\mathrm{HS}}$ dark matter gauge bosons. For values of the custodial symmetry breaking scale near to the Grand Unification scale, and for dark matter masses around $400 \mathrm{GeV}$ and below, this line could be in reach of sensitivity of the Fermi LAT gamma-ray line searches. On the other hand, a production of an observable amount of electrons and positrons or anti-protons is very model dependent. In most cases electrons and positrons are produced in the fragmentation of scalar or vector bosons and lead to a very flat spectrum. An interesting exception occurs for the operator case $\mathrm{D}$ which features two-body decay modes into lepton pairs. In this case the produced positron spectrum can rise more steeply, but, when also taking other observations into account, still not enough to explain the PAMELA observations alone. 

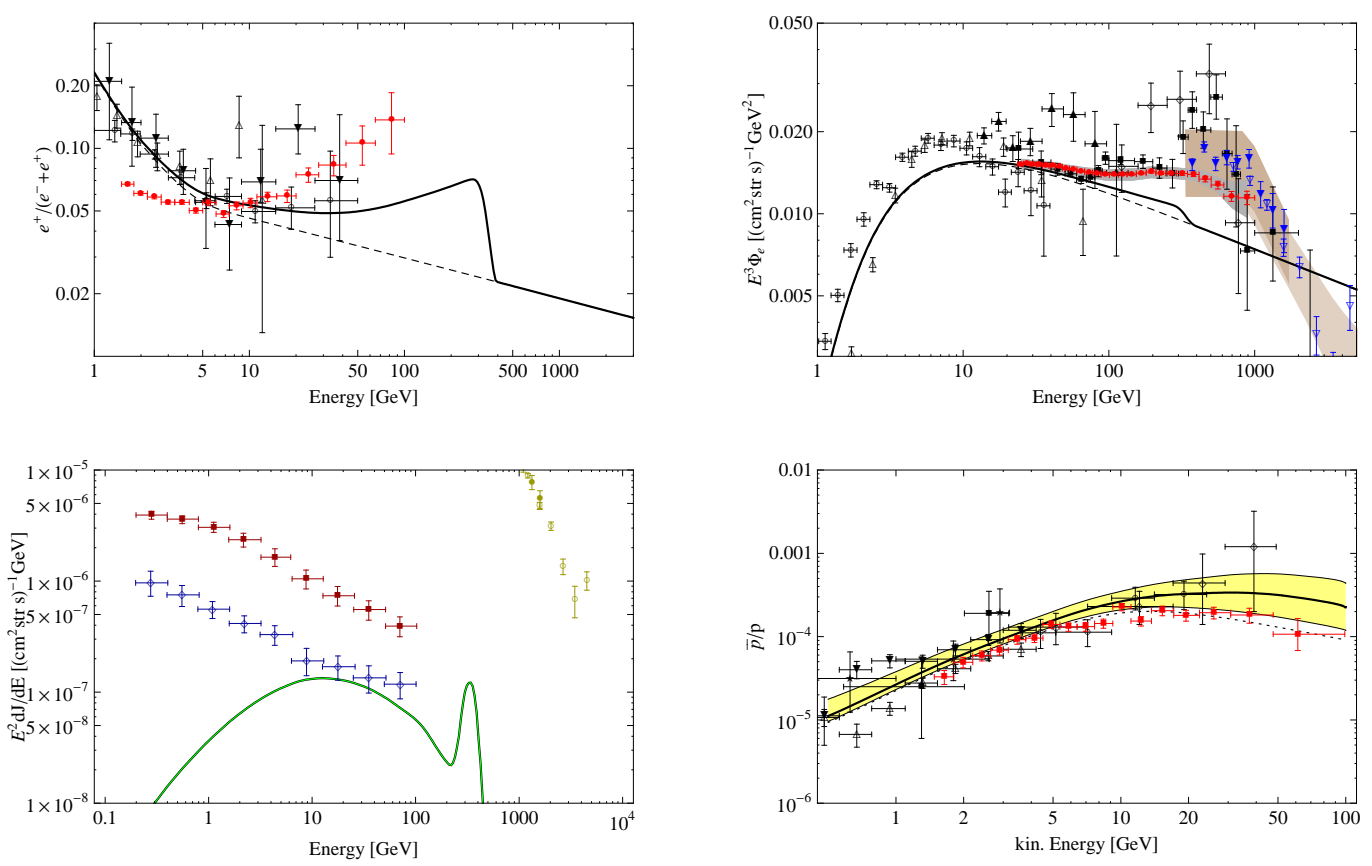

Figure 5: Like Fig. 1, but for case D, benchmark 2, with $\tau_{\mathrm{DM}}=6.7 \times 10^{26} \mathrm{~s}(\Lambda=$ $\left.1.5 \times 10^{16} \mathrm{GeV}\right)$.

\section{Effects of the annihilation processes with one dark matter particle in the final state}

The model considered above has the interesting and rather peculiar property that it allows annihilation processes with one dark matter particle in the final state, i.e. $A_{i} A_{j} \rightarrow$ $A_{k} \eta$ annihilations via an intermediate $A_{k}$, Fig. 2 of [1]. In ordinary models based on a $Z_{2}$ symmetry such processes are strictly forbidden, they would be equivalent to $Z_{2}$ breaking at the renormalizable level and therefore to fast DM decay. The non-abelian character of the custodial symmetry responsible for the stability of the hidden vectors allows these processes through the trilinear coupling $\mathcal{L} \ni-\frac{1}{4} F^{\mu \nu} F_{\mu \nu} \ni-\frac{1}{2} g_{\phi} \varepsilon_{i j k} A_{j}^{\mu} A_{k}^{\nu}\left(\partial_{\mu} A_{i \nu}-\partial_{\nu} A_{i \mu}\right)$. As pointed out in Refs. [1, 2] these "trilinear" processes do not bring nevertheless any new radical change in the freeze-out mechanism. In the Boltzmann equations (where $n=n_{1}+n_{2}+n_{3}$ is the density of $A$ states)

$$
\frac{d n}{d t}+3 H n=-\frac{\left\langle\sigma_{i i} v\right\rangle}{3}\left(n^{2}-n_{E q}^{2}\right)-\frac{\left\langle\sigma_{i j} v\right\rangle}{3} n\left(n-n_{E q}\right),
$$

these terms, parametrized by $\sigma_{i j}$, behave linearly in $n-n_{E q}$, whereas the ordinary annihilations, parametrized by $\sigma_{i i}$, behave linearly in $n^{2}-n_{E q}^{2}$. Since $n^{2}-n_{E q}^{2} \approx 2 n\left(n-n_{E Q}\right)$ near freeze-out, the relic abundance behaves as usual $\Omega_{D M} \propto 1 / \operatorname{Max}\left(\sigma_{i j}, 2 \sigma_{i i}\right)$. However these "trilinear" processes contribute with a rate expected to be similar to the one of 

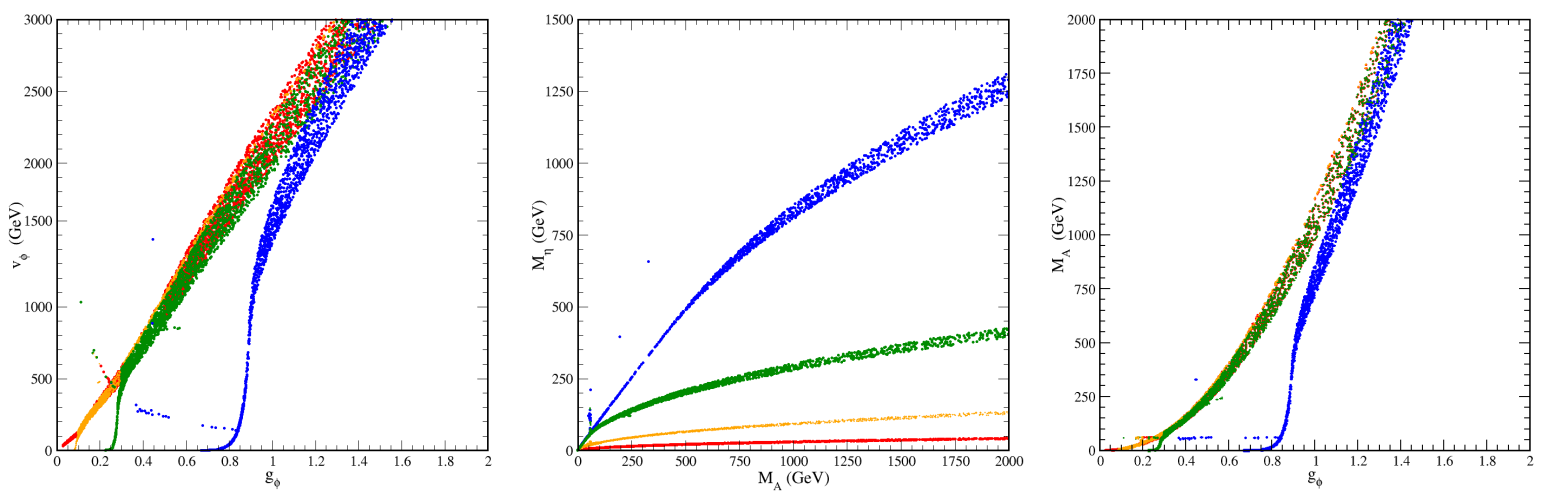

Figure 6: Parameter space leading to $0.091<\Omega h^{2}<0.129$ for the small $\lambda_{m}$ regime $\left(10^{-7}<\lambda_{m}<10^{-3}\right)$. From left to right: $v_{\phi}$ vs $g_{\phi}, M_{\eta}$ vs $M_{A}$ and $M_{A}$ vs $g_{\phi}$. The different curves are for various values of $\lambda_{\phi}: \lambda_{\phi}=10^{-4}$ (red), $\lambda_{\phi}=10^{-3}$ (orange), $\lambda_{\phi}=10^{-2}$ (green) and $\lambda_{\phi}=10^{-1}$ (blue). The Higgs mass is fixed at $M_{h}=120 \mathrm{GeV}$. The dots off the main "sequences" correspond to Higgs or $\eta$ resonant annihilations, for $M_{A}=g_{\phi} v_{\phi} / 2 \sim M_{h} / 2$ and $M_{\eta} / 2$ respectively.

the processes with no dark matter particle in the final states and consequently should be properly taken into account. This is what is done here, for "small" Higgs portal coupling, $\lambda_{m}<10^{-3}$, and for "large" Higgs portal coupling, $\lambda_{m}>10^{-3}$.

Considering first the small $\lambda_{m}$ regime, in Fig 6 are shown the values of the gauge coupling $g_{\phi}$ vs $v_{\phi}, M_{A}$ vs $M_{\eta}$ and $M_{A}$ vs $g_{\phi}$, which lead to a relic density within the WMAP range $0.091<\Omega h^{2}<0.129$ at $3 \sigma$ [68, in agreement with the direct detection experimental upper bounds from CDMS [69] and Xenon10 [70] (see Fig. 8 below). These graphs show corrections of order unity with respect to the corresponding result without the "trilinear" processes, Fig. 3 of Ref. [1]. The dominant processes are the annihilations $A_{i} A_{i} \rightarrow \eta \eta$ and $A_{i} A_{j} \rightarrow \eta A_{k}$ which, unless the $\lambda_{\phi}$ coupling is large, have cross-sections proportional to $g_{\phi}^{4} / M_{A}^{2}$, leading to a $M_{A} \propto g_{\phi}^{2}$ quadratic behavior in Fig. 6. The only exception to this behavior is given by the resonant cases, when $M_{A} \sim M_{h} / 2$ or $M_{A} \sim$ $M_{\eta} / 2$.

The corresponding plots for the large Higgs portal regime are given in Fig 7 . In this case the deviations due to the new $A_{i} A_{j} \rightarrow A_{k} \eta$ processes are more difficult to single out, since more annihilation channels (involving $\lambda_{m}$ ) contribute to the relic density. But with respect to the case already discussed in Fig. 4 of Ref. [1], one finds points with lighter $M_{\eta}$ and smaller $g_{\phi}$ for a same value of $v_{\phi}$. The plot $M_{A}$ vs $g_{\phi}$ indicates again that the freeze-out has a complicated dependence on the couplings of the model. Some of the dots still display the quadratic behavior of Fig. 6, when the dominant annihilation channels are $A_{i} A_{i} \rightarrow \eta \eta$ and $A_{i} A_{j} \rightarrow A_{k} \eta$. 

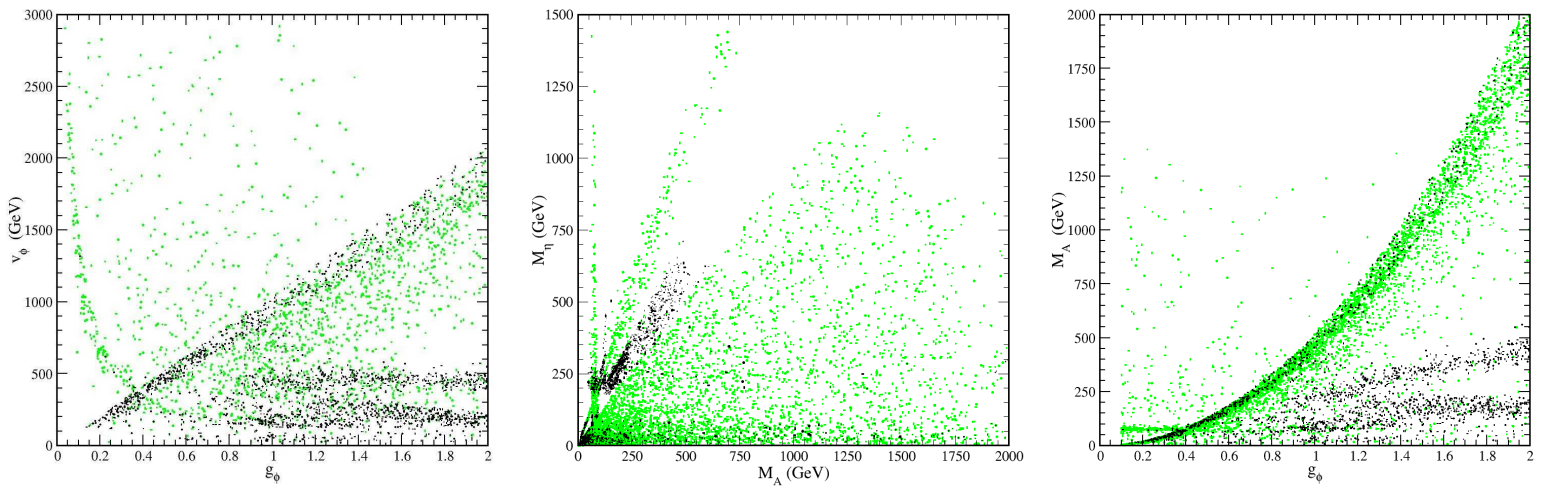

Figure 7: Parameter space leading to $0.091<\Omega h^{2}<0.129$ for the large $\lambda_{m}$ regime $\left(10^{-3}<\lambda_{m}<1\right)$, from left to right: $v_{\phi}$ vs $g_{\phi}, M_{\eta}$ vs $M_{A}$ and $M_{A}$ vs $g_{\phi} . \lambda_{\phi}$ is varied in the range $10^{-5}-1$, the Higgs mass between $M_{h}=114.4 \mathrm{GeV}$ and $M_{h}=160 \mathrm{GeV}$. Here too one can recognize the resonant cases, from both the Higgs and the $\eta$ bosons, for $M_{A}=g_{\phi} v_{\phi} / 2 \sim m_{h} / 2, m_{\eta} / 2$ respectively. All dots satisfy the LEP constraints on the $T$ parameter and on $h \rightarrow f \bar{f}$ decay. They also are in agreement with the direct detection bounds from CDMS [69] and Xenon [70. The black dots correspond to instances which lead to a spin-independent elastic cross-section at most one order of magnitude below these bounds.

At tree level the elastic scattering of the vector dark matter on a nucleon $n$ is spin independent, mediated by an $h$ or $\eta$ boson, and the full expression for the cross-section reads:

$$
\sigma^{S I}(A n \rightarrow A n)=\frac{1}{64 \pi^{2}} f^{2} g_{\phi}^{4} \sin ^{2} 2 \beta m_{n}^{2} \frac{v_{\phi}^{2}}{v^{2}} \frac{\left(M_{\eta}^{2}-M_{h}^{2}\right)^{2}}{M_{\eta}^{4} M_{h}^{4}} \frac{\mu_{n}^{2}}{M_{A}^{2}},
$$

with $\mu_{n}=m_{n} M_{A} /\left(m_{n}+M_{A}\right)$ the reduced mass and $m_{n}$ the nucleon mass. The parameter $f$ designs the Higgs nucleon coupling, $f \equiv\left\langle n\left|\sum_{q} m_{q} \bar{q} q\right| n\right\rangle$ and is taken to the value of $f=0.3$.

Imposing the relic density constraint, the predictions for the direct detection rate are given in Fig. 8, together with the upper bounds of CDMS 69, Xenon10 70, and the recent final results from CDMS-II [71]. In the small Higgs portal regime, $\lambda_{m} \lesssim 10^{-3}$, even though cross sections are suppressed by 2 powers of $\lambda_{m}$, large direct detection rates can be obtained for small $\lambda_{\phi}$ couplings because in this case $m_{\eta}<m_{h}$ and the $A n \rightarrow A n$ cross section scales as $\lambda_{m}^{2} / \lambda_{\phi}^{2}$, Eq. (4.2). For a dark matter mass from few GeV all the way up to the multi TeV range, a spin independent cross section of the order of the current experimental sensitivities can be obtained for values of $\lambda_{\phi}$ of order or below few $10^{-4}$. The values of the various parameters required in this case can be read off in Fig. 6 . The value of the cross-section does not depend on the dark matter mass if this mass is large, as indicated by the plateaux for different values of $\lambda_{\phi}$. For the large Higgs portal 

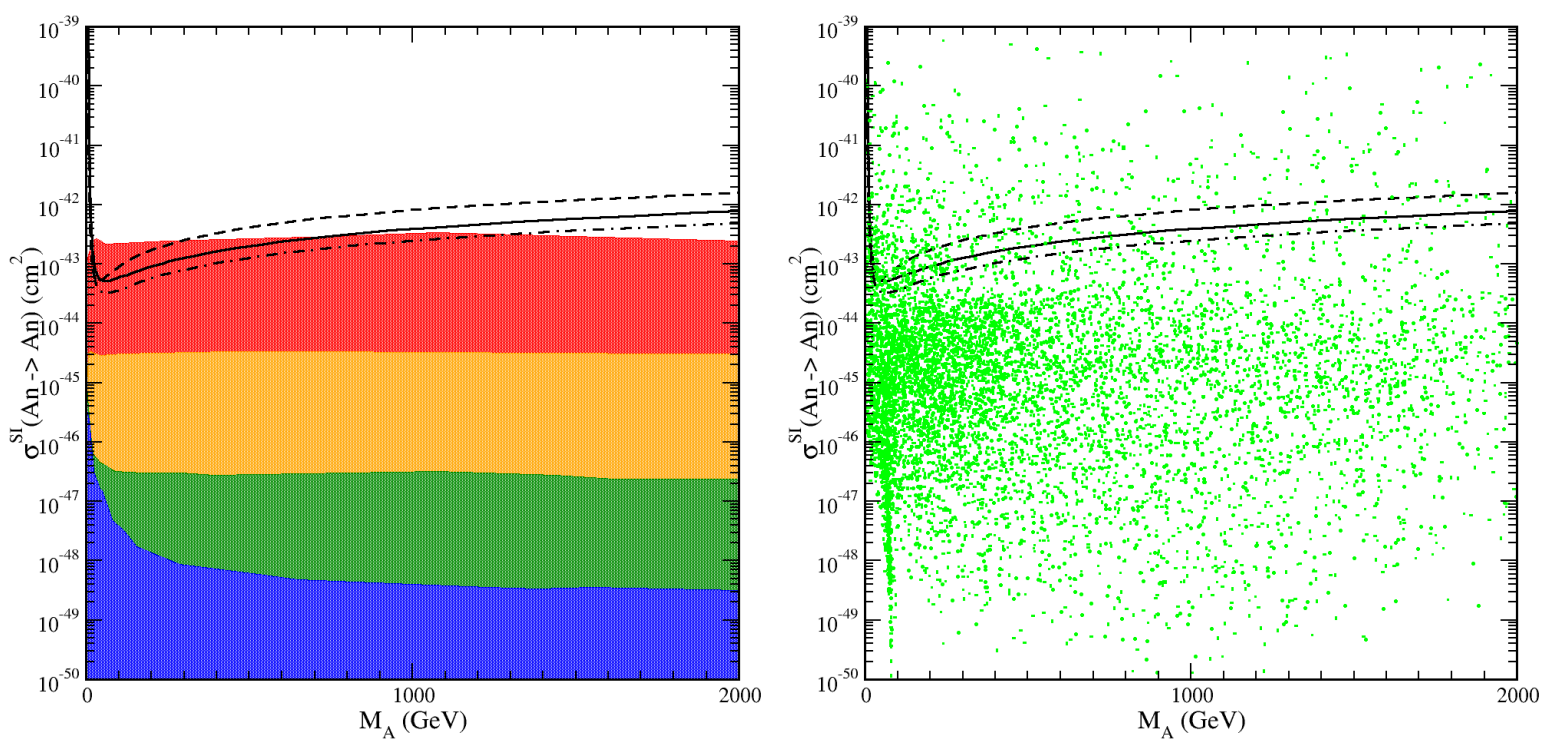

Figure 8: Obtained spin independent cross-section on nucleon $\sigma^{S I}(A n \rightarrow A n)$ versus $M_{A}$, in agreement with the constraint $0.091<\Omega h^{2}<0.129$. Small $\lambda_{m}$ regime $\left(10^{-7}<\lambda_{m}<\right.$ $10^{-3}$ ) on the left and large Higgs coupling portal, $\lambda_{m}>10^{-3}$ on the right. The color caption is as in Figs. 6 and 7. The thick (dashed) black curve is the CDMS (Xenon10) upper bounds at $90 \%$ C.L.. The dotted-dashed curve is the recent published CDMS-II upper bound, at $90 \%$ C.L.

regime, $\lambda_{m} \gtrsim 10^{-3}$, direct detection rates of order the present experimental sensitivity or exceeding it are easily produced. For illustration, among the sets of parameters that lead to the right relic density in Fig. 7, we have denoted by black dots the ones which lead to an elastic cross-section on nucleon at most one order of magnitude below the CDMS [69] and Xenon [70] limits. Here too a dark matter mass in the whole range from $1 \mathrm{GeV}$ up to few TeV can be accommodated. Even though other values are possible, the $\eta$ mass tends to be either small, below $100 \mathrm{GeV}$, or slightly larger than the dark matter mass. For $M_{A}$ larger than $\sim 700 \mathrm{GeV}$ one recovers the linear relation between $v_{\phi}$ and $g_{\phi}$ and the corresponding quadratic behavior of $M_{A}$ in $g_{\phi}$, indicating that the pure hidden sector annihilations driven by the $g_{\phi}$ coupling are dominant, as in the small Higgs portal regime.

Note that all the dots shown in the figures above satisfy the LEP constraints. The mixing of the $\eta$ boson with the standard model Higgs affects the electroweak precision observables. The main constraint on the model parameters comes from the $\mathrm{T}$ parameter, since the $\eta$ is a neutral scalar which mixes with the Higgs boson. We use the same cuts as in Ref. [1], that is to require that $T-T_{S M}$ is in the conservative range $-0.27 \mp 0.05$ from [72]. For $M_{\eta}<114.4 \mathrm{GeV}$ the branching ratio $\eta \rightarrow f \bar{f}$ should not exceed the LEP 
direct search bounds, leading to an upper value on the mixing angle $\sin ^{2} \beta$, see Fig. 10 of Ref [73]. This bound constraints more the large Higgs portal coupling regime which involve large mixing angles. In addition, we take into account the combined analysis of the CDF and DO collaborations, that excludes a Higgs in the mass range $160-170 \mathrm{GeV}$ at $95 \%$ C.L. [74].

\section{Conclusions}

We have shown in this paper that the vectors of a hidden, spontaneously broken, nonabelian gauge group constitute a viable dark matter particle which decays at cosmological times. Their longevity is due to an accidental custodial symmetry in the renormalizable Lagrangian. However, similarly to the proton, they are not expected to be absolutely stable due to the existence of non-renormalizable dimension six operators which induce the decay of the dark matter particle. We have identified the four dimension six operators which break the custodial symmetry and calculated the dominant decay modes. Taking advantage of the fact that the dark matter has spin-1, the gamma lines are produced at tree level from DM two-body decay which beside the $\gamma$ involve the standard model $h$ or hidden sector $\eta$ Higgs bosons. We have found that in all the cases an intense gamma-ray line is expected, which could be observed by the Fermi-LAT if the scale of custodial symmetry breaking is close to the Grand Unification scale. We have also calculated the positron fraction, total electron plus positron flux and the antiproton-to-proton fraction for these channels. Even though in these scenarios there is a sizable branching ratio into hadrons, the total antiproton-to-proton fraction is consistent with the measurements, while still producing an observable and possibly huge gamma-ray line. Finally we also improved the calculation of the relic density of hidden vectors including the dark matter annihilation processes with one dark matter particle in the final state. Direct detection rates close to the present limits can easily be produced for any dark matter mass within the GeV-multi TeV range.

\section{Acknowledgements}

The work of CA and TH is supported by the FNRS-FRS, the IISN and the Belgian Science Policy (IAP VI-11). The work of AI was partially supported by the DFG cluster of excellence "Origin and Structure of the Universe." 


\section{References}

[1] T. Hambye, Hidden vector dark matter, JHEP 01 (2009) 028, arXiv:0811.0172.

[2] T. Hambye and M. H. G. Tytgat, Confined hidden vector dark matter, Phys. Lett. B683 (2010) 39-41, arXiv:0907.1007.

[3] R. N. Mohapatra, New contributions to neutrinoless double-beta decay in supersymmetric theories, Phys. Rev. D34 (1986) 3457-3461.

[4] S. P. Martin, Some simple criteria for gauged R-parity, Phys. Rev. D46 (1992) 2769-2772, hep-ph/9207218.

[5] C. S. Aulakh, A. Melfo, A. Rasin, and G. Senjanovic, Supersymmetry and large scale left-right symmetry, Phys. Rev. D58 (1998) 115007, hep-ph/9712551.

[6] C. S. Aulakh, B. Bajc, A. Melfo, A. Rasin, and G. Senjanovic, SO(10) theory of R-parity and neutrino mass, Nucl. Phys. B597 (2001) 89-109, hep-ph/0004031.

[7] M. Kadastik, K. Kannike, and M. Raidal, Less-dimensions and matter parity as the origin of Dark Matter, Phys. Rev. D81 (2010) 015002, arXiv:0903.2475.

[8] M. Frigerio and T. Hambye, Dark matter stability and unification without supersymmetry, arXiv:0912.1545.

[9] PAMELA Collaboration, O. Adriani et. al., An anomalous positron abundance in cosmic rays with energies 1.5-100 GeV, Nature 458 (2009) 607-609, arXiv:0810.4995.

[10] I. V. Moskalenko and A. W. Strong, Production and propagation of cosmic-ray positrons and electrons, Astrophys. J. 493 (1998) 694-707, astro-ph/9710124.

[11] The Fermi LAT Collaboration, A. A. Abdo et. al., Measurement of the Cosmic Ray e+ plus e-spectrum from $20 \mathrm{GeV}$ to $1 \mathrm{TeV}$ with the Fermi Large Area Telescope, Phys. Rev. Lett. 102 (2009) 181101, arXiv:0905.0025.

[12] FERMI-LAT Collaboration, D. Grasso et. al., On possible interpretations of the high energy electron- positron spectrum measured by the Fermi Large Area Telescope, Astropart. Phys. 32 (2009) 140-151, arXiv:0905.0636.

[13] H.E.S.S. Collaboration, F. Aharonian et. al., The energy spectrum of cosmic-ray electrons at TeV energies, Phys. Rev. Lett. 101 (2008) 261104, arXiv:0811.3894. 
[14] H. E. S. S. C. F. Aharonian, Probing the ATIC peak in the cosmic-ray electron spectrum with H.E.S.S, Astron. Astrophys. 508 (2009) 561, arXiv:0905.0105.

[15] A. Ibarra and D. Tran, Decaying Dark Matter and the PAMELA Anomaly, JCAP 0902 (2009) 021, arXiv:0811.1555.

[16] A. Ibarra, D. Tran, and C. Weniger, Decaying Dark Matter in Light of the PAMELA and Fermi LAT Data, JCAP 1001 (2010) 009, arXiv:0906.1571.

[17] O. Adriani et. al., A new measurement of the antiproton-to-proton flux ratio up to 100 GeV in the cosmic radiation, Phys. Rev. Lett. 102 (2009) 051101, arXiv:0810.4994.

[18] D. Eichler, TeV particles as weakly unstable dark matter, Phys. Rev. Lett. 63 (1989) 2440.

[19] E. Nardi, F. Sannino, and A. Strumia, Decaying Dark Matter can explain the electron/positron excesses, JCAP 0901 (2009) 043, arXiv:0811.4153.

[20] A. Arvanitaki et. al., Astrophysical Probes of Unification, Phys. Rev. D79 (2009) 105022, $\operatorname{arXiv:0812.2075].}$

[21] K. Hamaguchi, S. Shirai, and T. T. Yanagida, Cosmic Ray Positron and Electron Excess from Hidden- Fermion Dark Matter Decays, Phys. Lett. B673 (2009) 247-250, arXiv:0812.2374.

[22] J. T. Ruderman and T. Volansky, Searching for Smoking Gun Signatures of Decaying Dark Matter, arXiv:0907.4373.

[23] J. T. Ruderman and T. Volansky, Decaying into the Hidden Sector, arXiv:0908.1570.

[24] L. Bergstrom and H. Snellman, Observable monochromatic photons from cosmic photino annihilation, Phys. Rev. D37 (1988) 3737-3741.

[25] S. Rudaz, On the annihilation of heavy neutral fermion pairs into monochromatic gamma-rays and its astrophysical implicaitons, Phys. Rev. D39 (1989) 3549.

[26] A. Bouquet, P. Salati, and J. Silk, $\gamma^{-}$ray lines as a probe for a cold dark matter halo, Phys. Rev. D40 (1989) 3168.

[27] F. Boudjema, A. Semenov, and D. Temes, Self-annihilation of the neutralino dark matter into two photons or a $Z$ and a photon in the MSSM, Phys. Rev. D72 (2005) 055024, hep-ph/0507127. 
[28] L. Bergstrom and P. Ullio, Full one-loop calculation of neutralino annihilation into two photons, Nucl. Phys. B504 (1997) 27-44, hep-ph/9706232.

[29] Z. Bern, P. Gondolo, and M. Perelstein, Neutralino annihilation into two photons, Phys. Lett. B411 (1997) 86-96, hep-ph/9706538.

[30] P. Ullio and L. Bergstrom, Neutralino annihilation into a photon and a $Z$ boson, Phys. Rev. D57 (1998) 1962-1971, hep-ph/9707333.

[31] L. Bergstrom, P. Ullio, and J. H. Buckley, Observability of gamma rays from dark matter neutralino annihilations in the Milky Way halo, Astropart. Phys. 9 (1998) 137-162, astro-ph/9712318.

[32] L. Bergstrom, T. Bringmann, M. Eriksson, and M. Gustafsson, Gamma rays from heavy neutralino dark matter, Phys. Rev. Lett. 95 (2005) 241301, hep-ph/0507229.

[33] M. Gustafsson, E. Lundstrom, L. Bergstrom, and J. Edsjo, Significant gamma lines from inert Higgs dark matter, Phys. Rev. Lett. 99 (2007) 041301, astro-ph/0703512.

[34] L. Bergstrom, T. Bringmann, M. Eriksson, and M. Gustafsson, Two photon annihilation of Kaluza-Klein dark matter, JCAP 0504 (2005) 004, hep-ph/0412001.

[35] C. B. Jackson, G. Servant, G. Shaughnessy, T. M. P. Tait, and M. Taoso, Higgs in Space!, arXiv:0912.0004.

[36] E. Dudas, Y. Mambrini, S. Pokorski, and A. Romagnoni, (In)visible Z' and dark matter, JHEP 08 (2009) 014, arXiv:0904.1745].

[37] W. Buchmuller, L. Covi, K. Hamaguchi, A. Ibarra, and T. Yanagida, Gravitino dark matter in R-parity breaking vacua, JHEP 03 (2007) 037, hep-ph/0702184.

[38] A. Ibarra and D. Tran, Gamma Ray Spectrum from Gravitino Dark Matter Decay, Phys. Rev. Lett. 100 (2008) 061301, arXiv:0709.4593.

[39] K. Ishiwata, S. Matsumoto, and T. Moroi, High Energy Cosmic Rays from the Decay of Gravitino Dark Matter, Phys. Rev. D78 (2008) 063505, arXiv:0805.1133.

[40] W. Buchmuller, A. Ibarra, T. Shindou, F. Takayama, and D. Tran, Probing Gravitino Dark Matter with PAMELA and Fermi, JCAP 0909 (2009) 021, arXiv:0906.1187]. 
[41] K.-Y. Choi, D. E. Lopez-Fogliani, C. Munoz, and R. R. de Austri, Gamma-ray detection from gravitino dark matter decay in the $\mu \nu S S M$, arXiv:0906.3681.

[42] J. F. Navarro, C. S. Frenk, and S. D. M. White, The Structure of Cold Dark Matter Halos, Astrophys. J. 462 (1996) 563-575, astro-ph/9508025.

[43] A. Ibarra, D. Tran, and C. Weniger, Detecting Gamma-Ray Anisotropies from Decaying Dark Matter: Prospects for Fermi LAT, arXiv:0909.3514. Accepted by PRD.

[44] K. Ishiwata, S. Matsumoto, and T. Moroi, Synchrotron Radiation from the Galactic Center in Decaying Dark Matter Scenario, Phys. Rev. D79 (2009) 043527, arXiv:0811.4492.

[45] J. Zhang et. al., Discriminating different scenarios to account for the cosmic e+ /- excess by synchrotron and inverse Compton radiation, Phys. Rev. D80 (2009) 023007, arXiv:0812.0522.

[46] L. Zhang, G. Sigl, and J. Redondo, Galactic Signatures of Decaying Dark Matter, JCAP 0909 (2009) 012, arXiv:0905.4952].

[47] M. Cirelli and P. Panci, Inverse Compton constraints on the Dark Matter e+eexcesses, Nucl. Phys. B821 (2009) 399-416, arXiv:0904.3830.

[48] K. Ishiwata, S. Matsumoto, and T. Moroi, Cosmic Gamma-ray from Inverse Compton Process in unstable Dark Matter Scenario, Phys. Lett. B679 (2009) 1-5, arXiv:0905.4593.

[49] V. L. Ginzburg, V. A. Dogiel, V. S. Berezinsky, S. V. Bulanov, and V. S. Ptuskin, Astrophysics of cosmic rays. Amsterdam, Netherlands: North-Holland (1990) 534 p.

[50] D. Maurin, F. Donato, R. Taillet, and P. Salati, Cosmic Rays below Z=30 in a diffusion model: new constraints on propagation parameters, Astrophys. J. 555 (2001) 585-596, astro-ph/0101231.

[51] A. M. Lionetto, A. Morselli, and V. Zdravkovic, Uncertainties of cosmic ray spectra and detectability of antiproton mSUGRA contributions with PAMELA, JCAP 0509 (2005) 010, astro-ph/0502406.

[52] L. J. Gleeson and W. I. Axford, Cosmic Rays in the Interplanetary Medium, Astrophys. J. 149 (Sept., 1967) L115. 
[53] HEAT Collaboration, S. W. Barwick et. al., Measurements of the cosmic-ray positron fraction from 1- GeV to 50-GeV, Astrophys. J. 482 (1997) L191-L194, astro-ph/9703192.

[54] A. W. Strong, I. V. Moskalenko, and O. Reimer, Diffuse continuum gamma rays from the Galaxy, Astrophys. J. 537 (2000) 763-784, astro-ph/9811296.

[55] G. Bertone, W. Buchmuller, L. Covi, and A. Ibarra, Gamma-Rays from Decaying Dark Matter, JCAP 0711 (2007) 003, arXiv:0709.2299.

[56] HEAT Collaboration, S. W. B. et al., Measurements of the cosmic-ray positron fraction from 1- GeV to 50-GeV, Astrophys. J. 482 (1997) L191-L194, astro-ph/9703192.

[57] M. Boezio et. al., The Cosmic-Ray Electron and Positron Spectra Measured at 1 AU during Solar Minimum Activity, Astrophys. J. 532 (Mar., 2000) 653-669.

[58] AMS-01 Collaboration, M. Aguilar et. al., Cosmic-ray positron fraction measurement from 1-GeV to 30- GeV with AMS-01, Phys. Lett. B646 (2007) 145-154, astro-ph/0703154.

[59] PPB-BETS Collaboration, S. Torii et. al., High-energy electron observations by PPB-BETS flight in Antarctica, arXiv:0809.0760.

[60] J. Chang et. al., An excess of cosmic ray electrons at energies of 300-800 GeV, Nature 456 (2008) 362-365.

[61] H. Matsunaga et. al., Measurement of low-energy cosmic-ray antiprotons at solar minimum, Phys. Rev. Lett. 81 (1998) 4052-4055, astro-ph/9809326.

[62] BESS Collaboration, S. Orito et. al., Precision measurement of cosmic-ray antiproton spectrum, Phys. Rev. Lett. 84 (2000) 1078-1081, astro-ph/9906426].

[63] M. Boezio et. al., The Cosmic-Ray Antiproton Flux between 0.62 and $3.19 \mathrm{GeV}$ Measured Near Solar Minimum Activity, Astrophys. J. 487 (Sept., 1997) 415.

[64] M. Boezio et. al., The Cosmic-Ray Antiproton Flux between 3 and $49 \mathrm{GeV}$, Astrophys. J. 561 (Nov., 2001) 787-799.

[65] J. W. Mitchell et. al., Measurement of 0.25-GeV to 3.2-GeV anti-protons in the cosmic radiation, Phys. Rev. Lett. 76 (1996) 3057-3060.

[66] M. Ackermann, "Observations of the isotropic diffuse gamma-ray emission with the Fermi LAT Telescope." Talk given at Fermi Symposium Nov 2009. 
[67] A. A. Abdo, M. Ackermann, and M. Ajello, Fermi LAT Search for Photon Lines from 30 to $200 \mathrm{GeV}$ and Dark Matter Implications, arXiv:1001.4836.

[68] WMAP Collaboration, E. Komatsu et. al., Five-Year Wilkinson Microwave Anisotropy Probe (WMAP) Observations:Cosmological Interpretation, Astrophys. J. Suppl. 180 (2009) 330-376, arXiv:0803.0547].

[69] CDMS Collaboration, Z. Ahmed et. al., Search for Weakly Interacting Massive Particles with the First Five-Tower Data from the Cryogenic Dark Matter Search at the Soudan Underground Laboratory, Phys. Rev. Lett. 102 (2009) 011301, arXiv:0802.3530.

[70] J. Angle et. al., Limits on spin-dependent WIMP-nucleon cross-sections from the XENON10 experiment, Phys. Rev. Lett. 101 (2008) 091301, arXiv:0805.2939].

[71] The CDMS Collaboration, Z. Ahmed et. al., Results from the Final Exposure of the CDMS II Experiment, arXiv:0912.3592.

[72] LEP EW WG plots for summer 2005 conferences (after LP05/EPS05).

[73] LEP Working Group for Higgs boson searches Collaboration, R. Barate et. al., Search for the standard model Higgs boson at LEP, Phys. Lett. B565 (2003) 61-75, hep-ex/0306033.

[74] CDF Collaboration, Combined CDF and DZero Upper Limits on Standard Model Higgs-Boson Production with up to 4.2 fb-1 of Data, arXiv:0903.4001. 